\title{
Influence of selective laser melting process parameters on texture evolution in pure copper
}

\author{
S. D. Jadhav ${ }^{a}$, S. Dadbakhsh ${ }^{b}$, L. Goossens ${ }^{b}$, J-P Kruth ${ }^{b}$, J. Van Humbeeck ${ }^{a}$, K. \\ Vanmeensel $^{\mathrm{a}}$
}

a Department of Materials Engineering, KU Leuven, Kasteelpark Arenberg 44, B-3001 Heverlee, Belgium

${ }^{\mathrm{b}}$ PMA, Department of Mechanical Engineering, KU Leuven \& Member of Flanders Make, Celestijnenlaan 300, B-3001 Heverlee, Belgium

* Corresponding author: E-mail address: suraj.jadhav@kuleuven.be

\begin{abstract}
In the present work, crack free pure copper parts with relative densities exceeding $98 \%$ are processed by selective laser melting (SLM) using a high-power fiber laser. The influence of different laser scan parameters such as hatch spacing, laser power and scan speed on the texture evolution in the manufactured parts is determined. On the top surfaces, a clear interrelationship between laser scan parameters and crystallographic texture is established. This shows that the texture at the top surface can be very strong especially when a high laser power and a low scanning speed is used. However, a random texture exists in the core of the sample due to successive re-melting and altered heat gradients induced by the application of a $90^{\circ}$ scan rotation between subsequent layers. A maximum electrical conductivity of $88 \%$ of the International Annealed Copper Standard (IACS) is achieved in the as built state. Damage to the optical mirror of the SLM machine is observed and recommendations for sustainable upscalability are proposed.
\end{abstract}

Keywords: Selective Laser Melting, SLM, Copper, Fiber Laser, Texture, Reflection, Reflectivity, Absorptance, Absorptivity

\section{1) Introduction}

Selective Laser Melting (SLM) is an additive manufacturing (AM) process wherein a high-power laser is allowed to interact with a metal powder bed. This causes fusion of the powder and subsequent welding with the substrate material. The process is repeated layer by layer and a 3D object is manufactured. SLM has important advantages over traditional manufacturing processes including effective material usage, flexible on-site manufacturing capacity and fabrication of complex, porous, hollow, fine features and conformal shapes. SLM allows to form complex parts with fine features providing larger surface area for heat transfer applications, such as heat exchangers and heat sinks (Aurubis, 2013). For example, (Neugebauer et al., 2011) has increased the heat exchange efficiency of an air-water heat exchanger using a combination of high thermal conductivity alloy such as AlSi10Mg (120-170 $\mathrm{W} /(\mathrm{m} . \mathrm{K}))$, and SLM as a manufacturing process. 
Despite the advantages offered by SLM to manufacture heat transfer objects with higher efficiency, the SLM materials palette is limited to a limited number of alloys such as Ti-6Al4V (7.2 W/(m.K)), Stainless steel 316L (15.2 W/(m.K)), Maraging steel $(25.5 \mathrm{~W} /(\mathrm{m} . \mathrm{K}))$, AlSi10Mg (120-170 W/(m.K)) (Thijs, 2014) Hence, one of the focal points of the SLM community is to extend the metal alloy palette with highly conductive metals such as copper (oxygen free copper: $398 \mathrm{~W} /(\mathrm{m} . \mathrm{K})$ ) and copper based alloys: 160-340 (W/(m.K)), which can provide significantly higher electrical and thermal conductivity values (Ryusuke et al., 2018).

The successful elaboration of fully dense copper parts by SLM, combining a high geometrical complexity and a high thermal/electrical conductivity would allow to manufacture complex shaped heat transfer objects with unforeseen heat transfer efficiency (Neugebauer et al., 2011). However, achieving crack-free and dense samples of pure copper using SLM is a challenge (Lykov et al., 2016). This is due to i) the very high laser reflectivity of copper when fiber lasers with a wavelength of $\sim 1080 \mathrm{~nm}$ (near Infra-red region) are used and ii) the high thermal conductivity of copper, leading to fast heat dissipation and concomitant stress built up (Trevisan et al., 2017). Due to these challenges, recent research efforts resulted in upgrading commercially available SLM machines, either by the implementation of a $400 \mathrm{~W}$ lasers with a shorter wavelength ( $515 \mathrm{~nm}$ ) (Heussen and Meiners, 2017) or alternatively by the use of a much stronger fiber laser with a laser power up to $1 \mathrm{~kW}$ (Ikeshoji et al., 2018). Regardless of all these efforts, to the best knowledge of the authors, successful production of highly conductive pure copper is still very limited and electrical conductivity values have not been reported for pure copper parts fabricated by SLM. Moreover, the microstructure and texture in SLM processed copper have not been reported.

In the current work, a high power $(1 \mathrm{~kW})$ fiber laser is used to fabricate cube shaped samples from pure copper achieving relative densities exceeding $98 \%$ and stable sample geometries. Texture analysis is performed to illustrate the influence of SLM parameters on texture evolution in pure copper. As pure copper exhibits an FCC crystal structure, the crystallographic findings related to the solidification texture observed on the top surfaces can be generalized and used for other highly conductive FCC metals such as gold and silver. The top surface analysis and electrical conductivity measurements are performed on the samples with stable geometry. The consequences of laser back reflection from copper surfaces on the process stability and up scalability are addressed.

\section{2) Materials and Methods}

Gas atomized copper powder with $99.95 \%$ purity was purchased from Sandvik Osprey Ltd. As shown in Figure 1, a majority of particles exhibit a spherical shape, along with a limited amount of irregularly shaped particles. Some satellite particles can also be observed attached to larger powder particles. The particle size distribution was obtained using a Beckman Coulter LS 13320 particle size analyzer in dry mode and the oxygen content was measured by the inert gas fusion technique. Flowability was measured by the dynamic angle of repose (AoR) technique (Van den Eynde et al., 2018).

Optical/Laser absorption measurements were carried out on a Lambda 950 UV/VIS spectrophotometer from PerkinElmer using spectralon ${ }^{\circledR}$ as a $100 \%$ reflective reference material. The optical absorption of the used copper powder bed is $34 \%$ at a wavelength of 1080 $\mathrm{nm}$ (Near-Infra-red region). While, the optical absorption of a bulk copper part ranges from 2- 
$6 \%$, depending on the surface roughness of the irradiated surface (Bergström, 2008). The higher optical absorption for the powder bed compared to the bulk part is due to multiple internal reflections and subsequent absorption of the optical light between separate powder particles within the powder bed. All the powder characteristics are summarized in table 1.
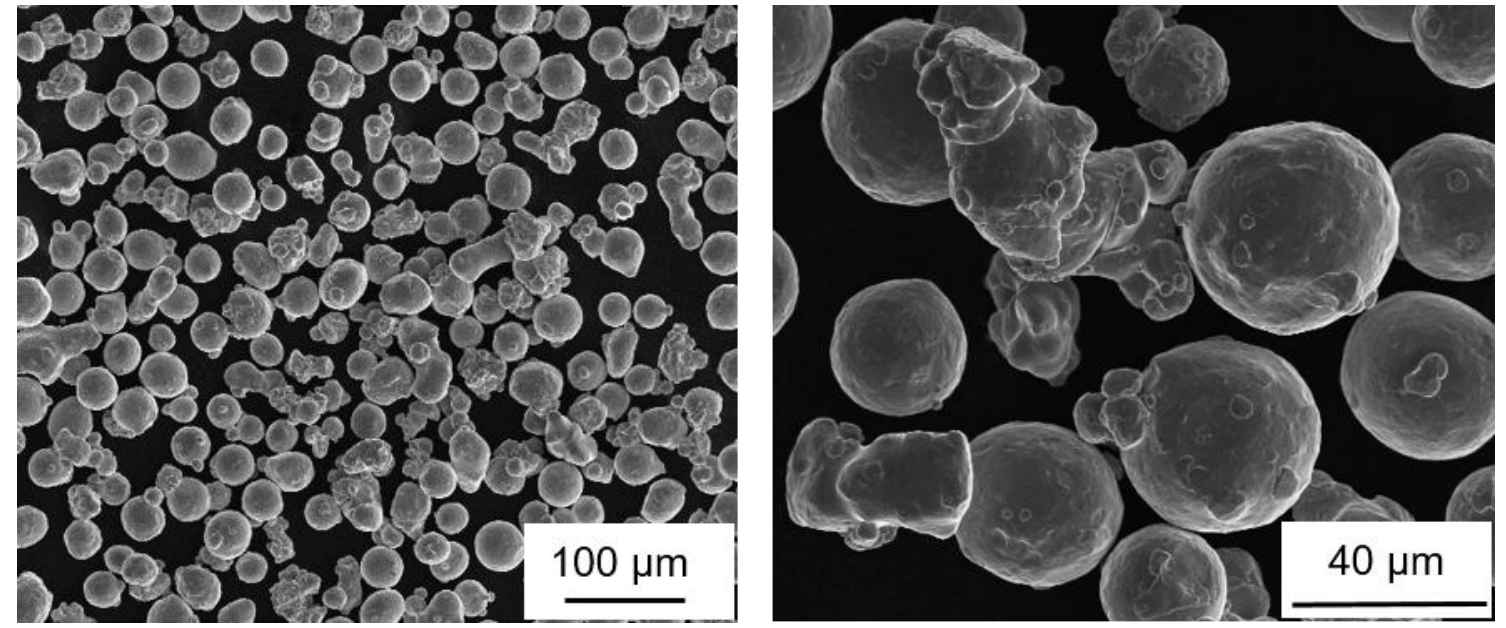

Figure 1: Scanning Electron Microscopy (SEM) image of the copper powder that was used within this study, showing spherical powder particles along with some irregular particles and a small fraction of satellite particles attached to larger copper powder particles.

Table 1: Powder characteristics of gas atomized copper powder used within this study.

\begin{tabular}{ll}
\hline Property & Reading \\
\hline Mean particle size $(\mu \mathrm{m})$ & $45 \pm 15$ \\
$\mathrm{~d}_{10}(\mu \mathrm{m})$ & 22.7 \\
$\mathrm{~d}_{50}(\mu \mathrm{m})$ & 34.8 \\
$\mathrm{~d}_{90}(\mu \mathrm{m})$ & 47.1 \\
Oxygen content $(\mathrm{ppm})$ & 542 \\
Optical/Laser absorption at 1080 nm $(\%)$ & 34 \\
Apparent and tapped density $(\%)$ & 49 and 56 \\
Average Angle of Repose $($ AoR $)$ & $30.1 \pm 0.8$ \\
\hline
\end{tabular}

STL file preparation was done using the Magics software package from Materialise, Belgium. SLM experiments to manufacture $10 \mathrm{~mm} \times 10 \mathrm{~mm} \times 10 \mathrm{~mm}$ cubes were carried out on an in-house developed SLM machine under an argon atmosphere. The oxygen level within the SLM processing chamber was kept below $100 \mathrm{ppm}$, in order to avoid any further oxidation of the copper powder. The SLM machine is equipped with a $1 \mathrm{~kW}$ single mode fiber laser with a wavelength of $1080 \mathrm{~nm}$ and a beam diameter of $40 \mu \mathrm{m}\left(1 / \mathrm{e}^{2}\right.$ value $)$ in a Gaussian intensity distribution profile.

Each layer was scanned once using a bi-directional (zig-zag) scan strategy and $90^{\circ}$ scan rotations were applied between successive layers. Different laser parameters (laser power $P$, scan speed $v$ and hatch spacing $h$ ) and a constant layer thickness of $30 \mu \mathrm{m}$ were applied. 
Volumetric laser energy densities $\left(\mathrm{J} / \mathrm{mm}^{3}\right)$ were calculated using equation (1) (Prashanth et al., 2017).

$E=\frac{P}{v * h * t} \quad$ - Equation 1

Where, $E$ is energy density $\left(\mathrm{J} / \mathrm{mm}^{3}\right), P$ is laser power $(\mathrm{W}), v$ is scan speed $(\mathrm{mm} / \mathrm{s}), h$ is hatch spacing $(\mathrm{mm})$, and $t$ is layer thickness $(\mathrm{mm})$.

The density of all samples was measured using the Archimedes method and relative densities are reported considering a theoretical density of copper as $8.93 \mathrm{~g} / \mathrm{cm}^{3}$. The microstructures of the as built top surfaces (XY plane) and polished cross-sectional surfaces (YZ plane) were visualized using scanning electron microscopy (SEM) equipped with a field emission gun (FEG) from Philips XL30 FEG, Eindhoven, The Netherlands. All samples were etched using $3.5 \mathrm{~g} \mathrm{FeCl}_{3}, 2.5 \mathrm{ml} \mathrm{HCl}$ and $75 \mathrm{ml} \mathrm{C}_{2} \mathrm{H}_{5} \mathrm{OH}$.

Texture analysis was carried out using a Bruker D8 X-ray diffraction (XRD) machine, equipped with a goniometer and a copper K-alpha X-ray radiation source with a wavelength of $1.54184 \AA$. All texture measurements were carried out on the flat surface of the samples. An $\mathrm{X}$-ray beam collimator with a diameter of $1 \mathrm{~mm}$ was used to ensure a high signal to noise ratio. For example: texture measurements for the sample processed using laser power of $800 \mathrm{~W}$, scan speed of $400 \mathrm{~mm} / \mathrm{s}$ and hatch spacing of $0.09 \mathrm{~mm}$ showed the $\langle 110\rangle$ peak intensities between 2700 to 5300 counts, while the background was measured at 300 counts. Pure copper powder was used as a reference, texture free material and the measured texture data was normalized with respect to the texture free copper powder after subtracting the background noise. Texture intensity is represented as a normalized intensity count equaling to the ratio of the texture intensity in the SLM processed part and the texture free copper powder. The pole figures and inverse pole figures were plotted using the MTEX open source software toolbox (Bachmann et al., 2010).

Texture studies were performed on three different sample surfaces, as shown schematically in Figure 2 using dashed lines. The scanning direction (SD) of the final layer and building direction (BD) of the cube shaped sample were parallel to the $\mathrm{X}$ and $\mathrm{Z}$ axis, respectively. Texture was measured on the top surface, identified as the XY-TOP plane. Moreover, the texture measurements were performed on a horizontal section parallel to the top surface near the center of the sample, labelled as the XY-MID plane. For XY-TOP and XY-MID planes, $\mathrm{BD}$ is located at the center of the pole figure pointing out of the plane. Texture measurements were also performed on a vertically oriented side plane, as indicated in Figure 2 (c), labelled as $\mathrm{YZ}$ plane. The data generated from the $\mathrm{YZ}$ plane were rotated $90^{\circ}$ around the $\mathrm{Y}$ axis in order to obtain BD again at the center of the pole figure orienting out of the plane. This enables visualization of all the pole figures from different planes within the same sample reference frame. 


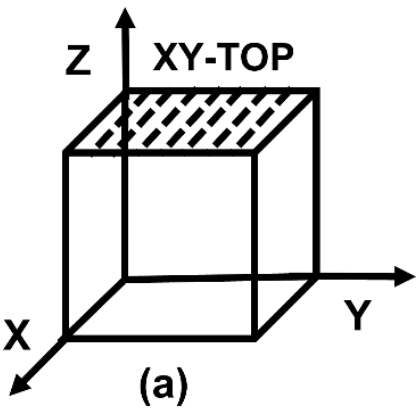

(a)

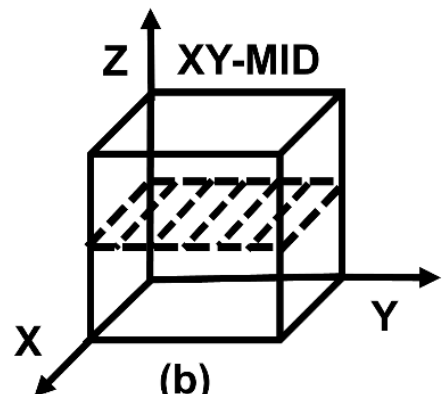

(b)

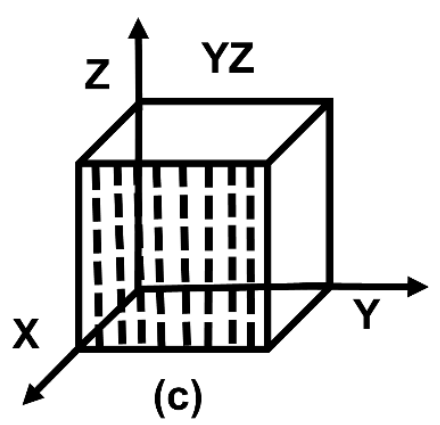

(c)

Figure 2: Schematic of texture measurement planes (a) top surface (XY-TOP), (b) horizontal section at center (XY-MID) and (c) vertical side surface - YZ plane. The measurement data for the $\mathrm{YZ}$ plane are rotated $90^{\circ}$ around the $\mathrm{Y}$ axis, in order to obtain BD parallel to $\mathrm{Z}$, similar to the case of XY-TOP and XY-MID planes.

Electrical conductivity values were obtained using a Burster, Resistomat ${ }^{\circledR}$ model 2301V001, applying a four-point measurement technique and an average of eight measurements were reported. This equipment is capable of measuring very low resistance values down to 0.1 $\mu \Omega$. 


\section{3) Results}

The aim of this research is to SLM dense and crack-free samples from pure copper in order to understand the role of solidification on microstructure and texture evolution. Therefore, the SLM process optimization will be discussed at first. Then, the solidification morphology observed in the top melt pool layer will be linked to the crystallographic texture observed on the top surface (XY-TOP) of cube shaped samples for different scan conditions. The bulk crystallographic texture and electrical conductivity measurements will be reported to determine the extent of anisotropy in the samples processed by SLM. Finally, the interrelationship between the generated solidification morphology and resulting crystallographic texture will be discussed.

\section{1) Determination of optimum SLM processing window}

Initially, cube shaped samples were fabricated using a laser power of $300 \mathrm{~W}$, laser scan speeds of 400 to $2500 \mathrm{~mm} / \mathrm{s}$ and hatch spacing of $0.05 \mathrm{~mm}$. A maximum relative density of $84 \%$ was realized for the sample processed at an applied energy density of $500 \mathrm{~J} / \mathrm{mm}^{3}$ (laser scan speed of $400 \mathrm{~mm} / \mathrm{s}$ ). Figures 3 (a) and 3 (b) show SEM images of the polished top surface (XY plane) and cross-section (YZ plane) of the sample, respectively. Even the sample with the highest relative density exhibited a very high fraction of remaining pores and therefore they could not be used for the texture measurements. Accordingly, a new SLM experiment was performed by applying higher laser powers/energy densities.

The samples were processed by varying the laser power input between 600 and $800 \mathrm{~W}$, the scan speed between 200 and $600 \mathrm{~mm} / \mathrm{s}$ and the hatching spacing between 0.07 and 0.09 $\mathrm{mm}$. Besides density measurements, the geometry and morphology of the laser melt tracks at the top surface was investigated and the potential consequence of the laser back reflection is reported.

Figure 3 (c) shows an image of the SLM parts along with the applied sets of process parameters. As can be seen in Figure 3 (c), only a narrow SLM processing window, allowing to produce dense cube shaped parts with stable geometries could be defined. Herein, a stable sample geometry refers to the cube shaped samples $(10 \mathrm{~mm} \times 10 \mathrm{~mm} \times 10 \mathrm{~mm})$ having flat surfaces that was fabricated with dimensional tolerance of $\pm 0.6 \mathrm{~mm}$ (measured using Vernier caliper) compared to original CAD design. The relative density values of these parts are plotted in Figure 3 (d) along with the applied energy density. The highest relative density values, exceeding $98 \%$ of the theoretical copper density, were achieved when energy densities in the range of $740-1120 \mathrm{~J} / \mathrm{mm}^{3}$ were applied. This was realized using i) a laser power $(P)$ of 600 W combined with a scanning speed $(v)$ of 200 and $300 \mathrm{~mm} / \mathrm{s}$ and a hatch spacing $(h)$ of 0.09 $\mathrm{mm}$ and ii) a laser power of $800 \mathrm{~W}$ combined with a scanning speed of $400 \mathrm{~mm} / \mathrm{s}$ and a hatch spacing of 0.07 and $0.09 \mathrm{~mm}$. The encircled data represents, the parts that did not delaminate from the base plate during SLM processing and exhibit a stable geometry. At higher energy densities above $1120 \mathrm{~J} / \mathrm{mm}^{3}$, a further decrease of the relative density values was observed.

Taking into account the optical properties of a copper powder bed and a solid copper substrate and bearing in mind the extremely high applied laser power and laser energy density in the current SLM experiment, the quality of the optical mirror incorporated within the used SLM machine was regularly checked. After SLM processing of the aforementioned cube 
shaped copper samples, a small scratch was observed in the dielectric coating that covered the optical mirrors.

(a)

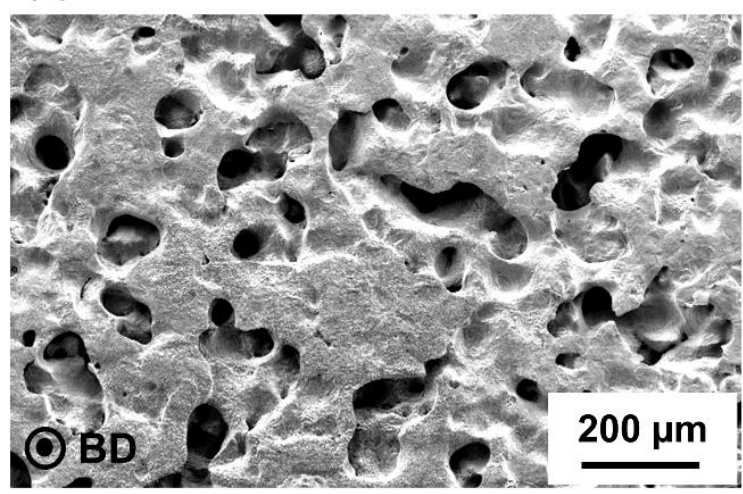

(c)

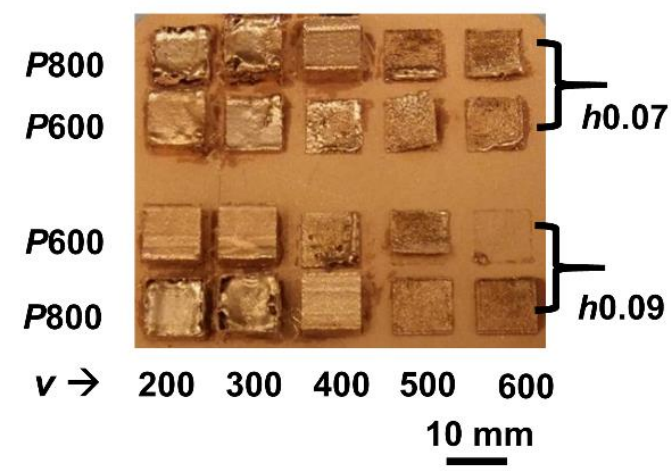

(b)

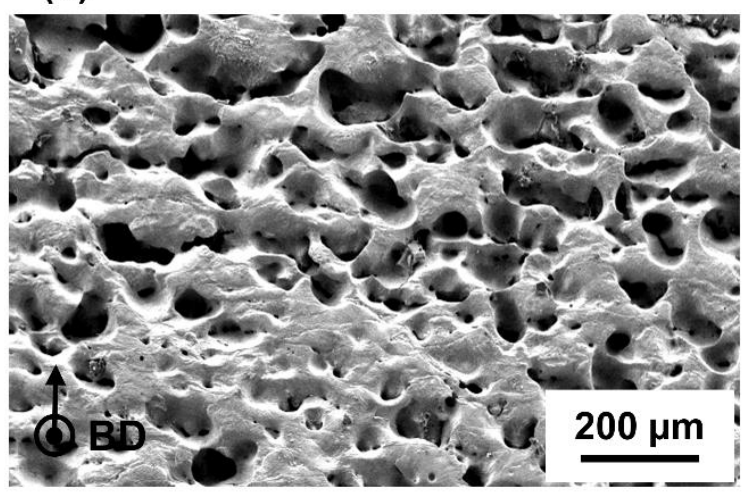

(d)

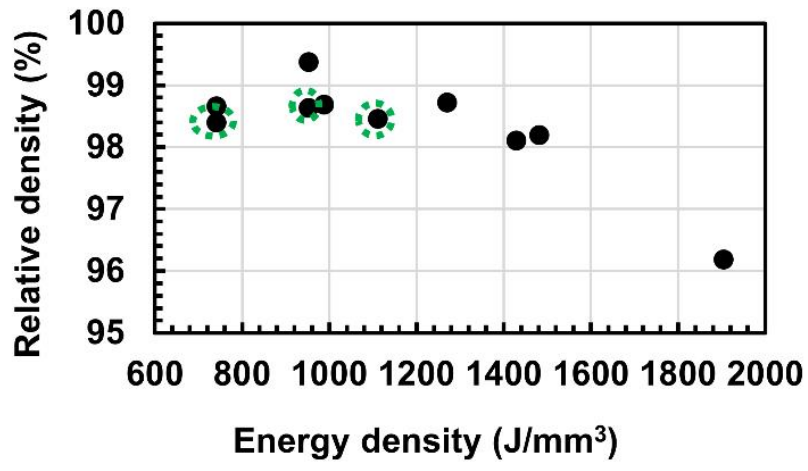

Figure 3: (a) and (b) SEM images for the polished top surface (XY plane) and cross-section of the sample processed at low power and energy density of $300 \mathrm{~W}$ and $500 \mathrm{~J} / \mathrm{mm}^{3}$, respectively. The sample shows a high porosity content consequently, it was not useful for the texture measurements, (c) Visual impression of the fabricated cube shaped parts along with the corresponding SLM process parameters $-P$ in Watt, $h$ in $\mathrm{mm}$ and $v$ in $\mathrm{mm} / \mathrm{s}$, (d) Plot of obtained relative density $(\%)$ of parts with respect to applied energy densities $\left(\mathrm{J} / \mathrm{mm}^{3}\right)$. Parts with a stable geometry are marked with a circle.

Figure 4 shows SEM images taken from the top surface of the samples. The influence of hatch spacing, power and scan speed on the geometry of melt tracks was investigated. Overall, all the geometrically stable parts have stable melt tracks without surface porosities. A mirroring effect of the melt pool boundaries along the fusion line was observed due to the applied bi-directional scanning strategy, as indicated by white dashed arrows. The samples produced using a laser power of $800 \mathrm{~W}$ show more than $50 \%$ overlap between neighboring melt tracks, whereas, melt tracks produced with a laser power of $600 \mathrm{~W}$ exhibit significantly less melt track overlap, as indicated by comparing Figures 4 (a) and 4 (b). When comparing Figures 4 (c) and 4 (d), an increased melt track overlap was observed when the hatch spacing was reduced. An increase in scan speed made geometry of the melt pool shape evolve from elliptical towards elongated elliptical or conical, as observed in a plane parallel to SD. Black arrows indicated in Figures 4 (a) and 4 (d) compare the distance from the tip to the approximate center of the melt pool. Simultaneously, within a YZ plane, this increase in scan speed will 
induce a reduction in the melt pool depth and hence shallower melt pools will be obtained. Additionally, the use of higher laser power will mainly deepen the melt pool along with a slight increase in the melt pool width.
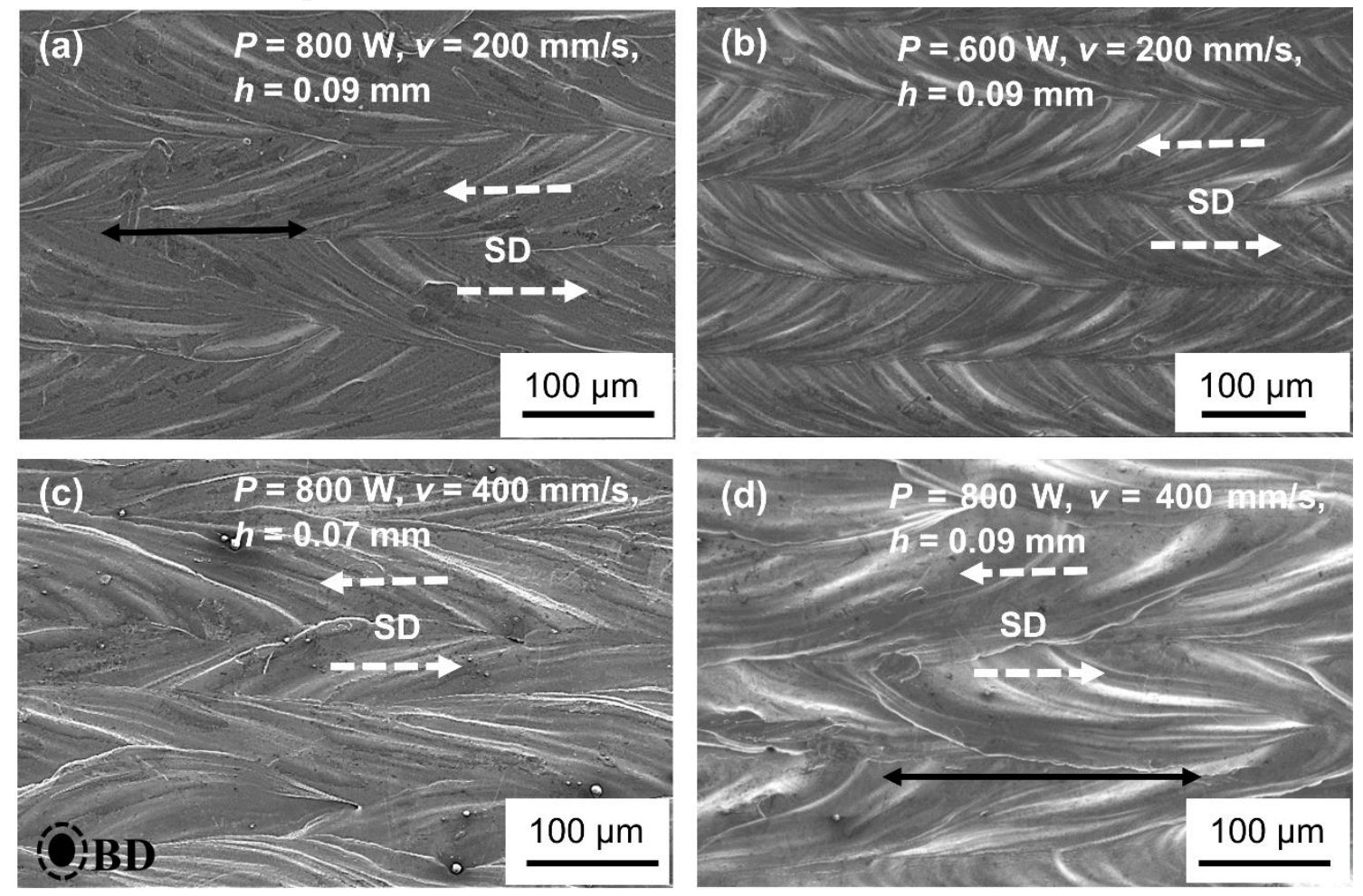

Figure 4: SEM image of sample top surfaces (XY-TOP) processed with different SLM scan parameters. The laser scan direction is indicated by the white dashed arrows. All samples are dense, crack-free and have stable laser melt tracks.

\section{2) Microstructural characterization of the top layer}

Figure 5 shows microstructures of the final laser scan tracks (non re-melted layer) for different SLM scan parameters in a YZ plane. Figures 5 (a), 5 (b) and 5 (c) show the microstructure obtained for samples processed with higher energy densities i.e. a power of 600 or $800 \mathrm{~W}$, a scan speed of $200 \mathrm{~mm} / \mathrm{s}$ and a hatch spacing of $0.09 \mathrm{~mm}$. The use of such a high power laser scanning at slower speed resulted in very deep melt pools, so that only the upper part of these melt pools (flat surface of the melt pool boundary as shown by black line in Figure 5 (c)) can be seen at selected magnification. Mainly, a planar solidification morphology is observed along with limited amount of regions exhibiting a cellular solidification morphology. The cells are oriented parallel to the XY-TOP plane as indicated by the white arrow in Figure 5 (c) or pointing out of the plane (encircled by white dashed ellipse). The solidification morphology for samples fabricated using high energy density demonstrates that the temperature gradient $(\mathrm{G})$ was high and parallel to the XY-TOP plane, as shown by black arrows perpendicular to the melt pool border and also pointing out of the plane (not shown, perpendicular to YZ). The interrelationship between solidification morphology and temperature gradient is elaborated in the discussion section (4.2).

Figures 5 (d), 5 (e) and 5 (f) show the solidification morphology of samples processed at a laser power of $800 \mathrm{~W}$, a scan speed of $400 \mathrm{~mm} / \mathrm{s}$ and a hatch spacing of 0.09 or $0.07 \mathrm{~mm}$ 
i.e. samples processed using relatively low energy density. At lower energy density and faster scan speed, the melt pools are less deep and elliptical in shape. Therefore, a curved surface of the melt pool is visible at these magnifications (as shown using black line in Figure 5 (f)). When looking in detail at the solidification morphology in the top melt pool layer, the solidification front is predominantly cellular, with the cells growing at an angle of $10-45^{\circ}$ with respect to the top surface. This observation demonstrates that the maximum temperature gradient is low and is inclined at similar angles with respect to the top surface. Typically, it is parallel and opposite to the orientation of the cells as shown in Figure 5 (f) by black arrows.

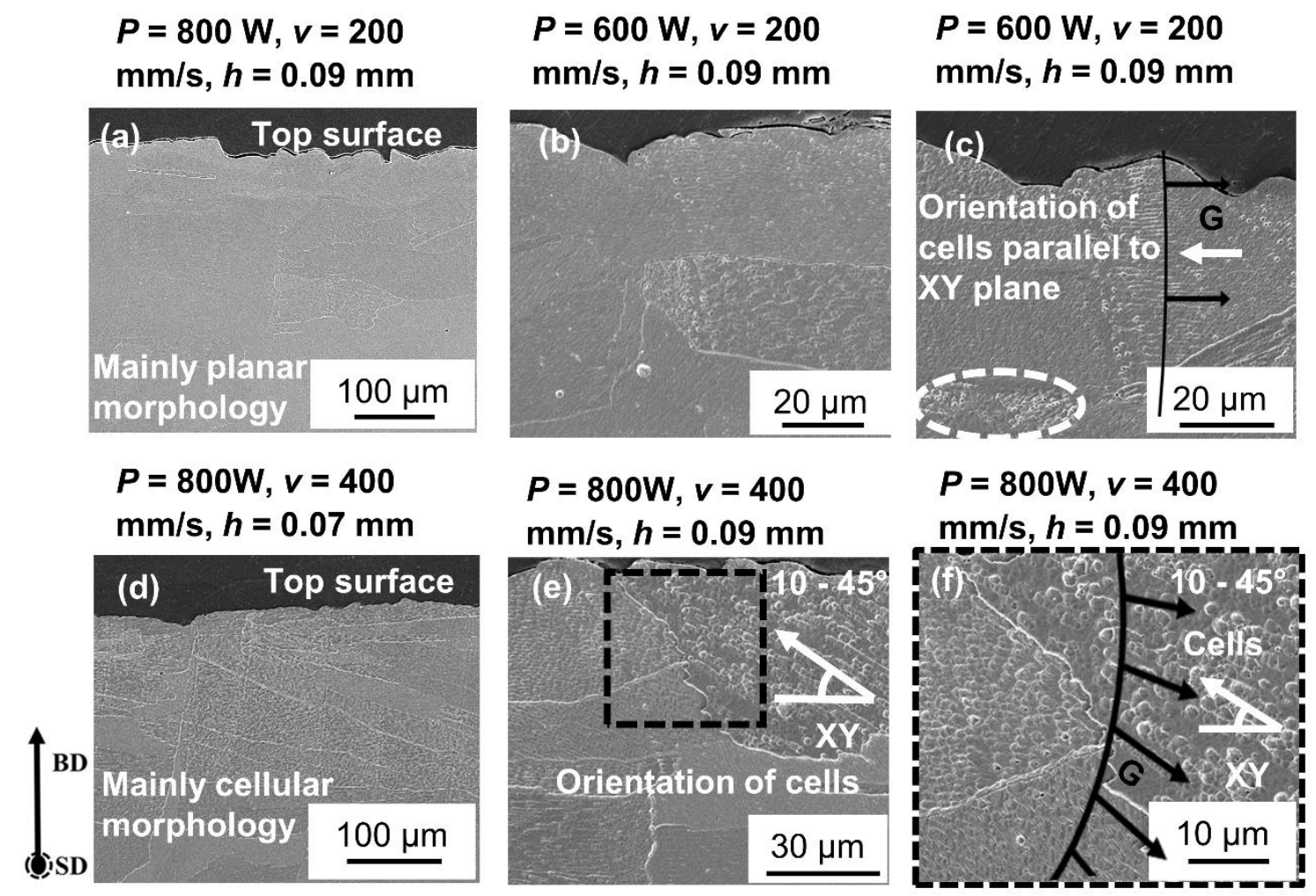

Figure 5: (a), (b) and (c) Microstructures of the melt pool in the sample top layer showing mainly planar solidification morphology along with some cells oriented parallel to XY-TOP plane. These samples were fabricated using high energy densities. (d), (e) and (f) Microstructures of the samples processed at lower energy densities showing predominantly cellular morphology in the top layer melt pool. Most of the cells are oriented at an angle of $10-45^{\circ}$ relative to the top surface as shown in Figure (e) and (f) by white arrows. The maximum temperature gradient $(\mathrm{G})$ is indicated using black arrows.

\section{3) Crystallographic characterization of the top surface (XY-TOP)}

After analyzing the solidification morphology in the top melt pool layer for samples processed with different laser parameter sets, crystallographic texture measurements were carried out on the sample top surfaces (XY-TOP). Texture analysis was performed to understand the influence of the employed laser settings on the solidification morphology and the texture development inside the top melt pool layer.

The X-ray diffraction pattern of copper shows $\langle 111\rangle,\langle 100\rangle,\langle 110\rangle$ and $\langle 113\rangle$ diffraction peaks up to $2 \theta=90^{\circ}$. During texture measurements it was observed that, depending 
on the applied SLM scan parameters, either the $\langle 100\rangle$ or $\langle 110\rangle$ direction was oriented parallel to the BD as shown by dashed black arrows in Figure 6. Therefore, only the $\{100\}$ and $\{110\}$ pole figures will be shown (where BD is in the center) for each varied scan parameters and interpretation of texture will be carried out considering BD of the sample as a reference frame.

The penetration depth of X-ray radiations inside copper is less than $50 \mu \mathrm{m}$ and the melt pool depths are over $100 \mu \mathrm{m}$. Therefore, the obtained texture information from the top surface only belongs to the upper part of the final melt pool layer (XY-TOP). The influence of the three varied SLM scan parameters such as hatch spacing, laser scan speed and laser power, on the crystallographic texture development within the final layer of the SLM sample will be described in the following paragraphs.

\subsection{1) Influence of hatch spacing}

Figures 6 (a) and 6 (b) show $\{100\}$ and $\{110\}$ pole figures measured on the top surface (XY-TOP) for the SLM parts processed at $P$ of $800 \mathrm{~W}, v$ of $400 \mathrm{~mm} / \mathrm{s}$ and a different hatch spacing of 0.07 and $0.09 \mathrm{~mm}$. The scanning direction for the top layer and the building direction (pointing out of the plane) are indicated next to the pole figure.

For both the SLM parameters, the pole figure shows a weak $<110>$ texture parallel to BD as indicated by black dashed arrows. However, the intensity of the $\langle 110\rangle$ texture is slightly reduced from 3.1 to 2.6 when increasing the hatch spacing from 0.07 to $0.09 \mathrm{~mm}$. The $\{100\}$ pole figure shows the presence of $\langle 100\rangle$ oriented grains parallel to SD (Scanning Direction) and TD (Transverse Direction) for both the cases. However, with a decrease of hatch spacing from 0.09 to $0.07 \mathrm{~mm}$, the <100> texture intensity at SD has slightly shifted towards BD.

\subsection{2) Influence of laser scan speed}

At an applied laser power of $800 \mathrm{~W}$ and a hatch spacing of $0.09 \mathrm{~mm}$, the influence of the laser scan speed was investigated for samples processed using scan speeds of 200 and 400 $\mathrm{mm} / \mathrm{s}$ as shown in Figures 6 (c) and 6 (b), respectively. The sample that was processed at a lower scan speed of $200 \mathrm{~mm} / \mathrm{s}$ shows a very strong texture (maximum normalized intensity count of 26 ) with <100> oriented grains parallel to BD (Figure 6 (c)). However, this strong $<100\rangle$ texture parallel to BD is diminished and a weak $\langle 110\rangle$ texture parallel to BD was obtained when a scan speed of $400 \mathrm{~mm} / \mathrm{s}$ was applied as indicated by the black dashed arrow in Figure 6 (b)). Thus, a clear interrelationship was found between the applied scan speed and the observed crystallographic texture obtained within the melt pool.

\subsection{3) Influence of laser power}

Figures 6 (c) and 6 (d) show a very strong influence of the applied power on the texture evolution, when a constant scan speed and hatch spacing of $200 \mathrm{~mm} / \mathrm{s}$ and $0.09 \mathrm{~mm}$, respectively, were applied. A power increase from 600 to $800 \mathrm{~W}$ has not only intensified the $<100>$ texture parallel to BD (maximum normalized intensity count of 26), but also changed the orientation of the $\langle 100\rangle$ orientated grains. The orientation of $\langle 100\rangle$ grains has changed from parallel to both SD and TD (Figure 6 (d) towards nearly $45^{\circ}$ inclined with respect to SD and TD (Figure 6 (c)), when the laser power was increased from 600 to $800 \mathrm{~W}$. 
(a) $P=800 W, v=400 \mathrm{~mm} / \mathrm{s}, h=0.07 \mathrm{~mm}$

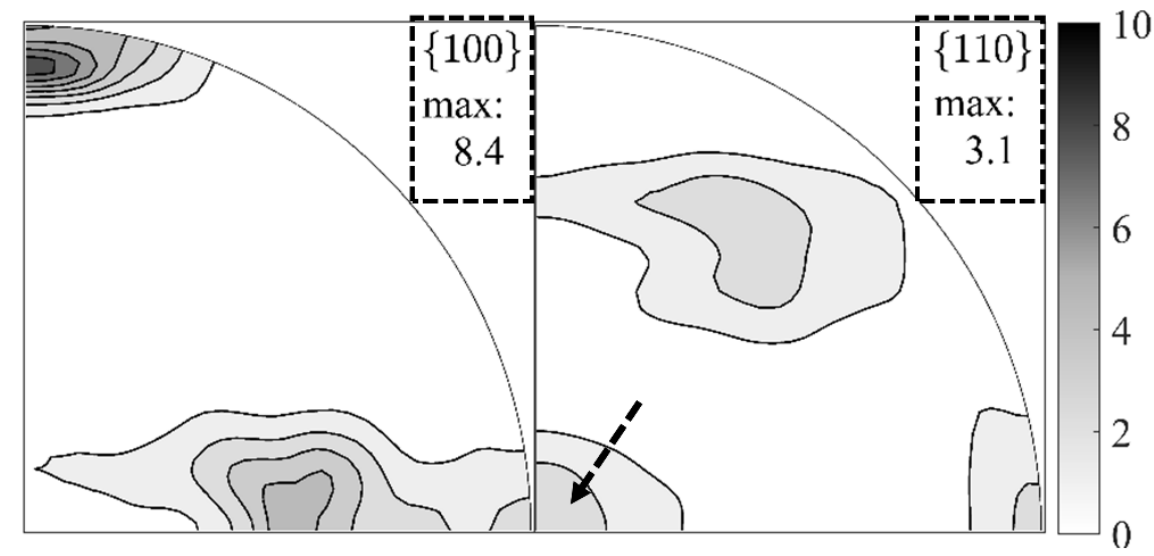

(b) $P=800 W, v=400 \mathrm{~mm} / \mathrm{s}, h=0.09 \mathrm{~mm}$

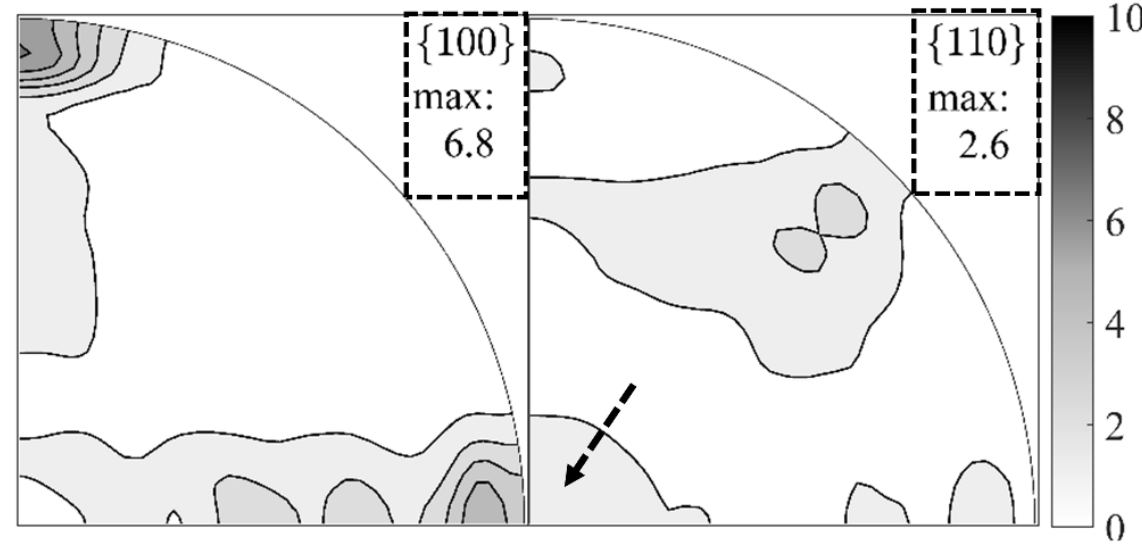

(c) $P=800 W, v=200 \mathrm{~mm} / \mathrm{s}, h=0.09 \mathrm{~mm}$

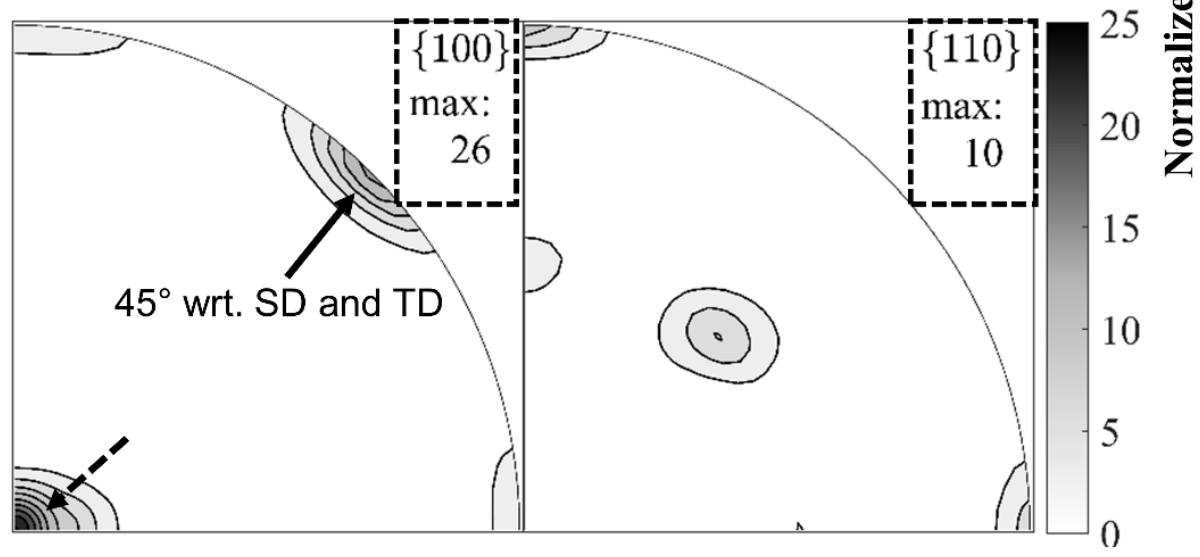

(d) $P=600 \mathrm{~W}, v=200 \mathrm{~mm} / \mathrm{s}, h=0.09 \mathrm{~mm}$
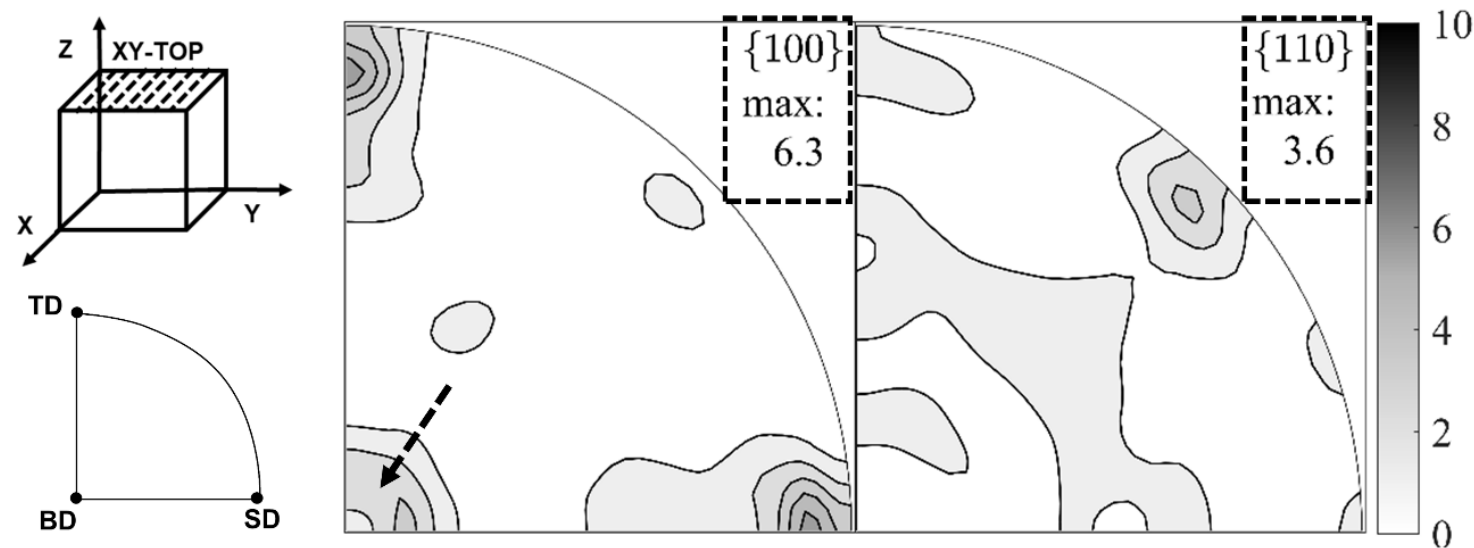
Figure 6: The $\{100\}$ and $\{110\}$ pole figures showing the influence of different SLM scan parameters on texture development within the final melt pool layer. The respective scanning parameters are indicated on the top of each pole figure and texture measurements were performed on the top surface (XY-TOP). Note that Figure 6 (c) has a different scale bar, since it shows a much stronger texture. The black dashed arrow indicates the texture type parallel to building direction. It is either $\langle 100\rangle$ or $\langle 110\rangle$ parallel to the building direction depending on the employed laser setting.

\section{4) Crystallographic characterization of the samples beneath the top layers (YZ)}

In the previous section, the influence of the laser scan parameters on the texture evolution within the top surface of the sample was evaluated. However, to evaluate the effect of re-melting and heat exposure between the layers, it is necessary to characterize the re-melted layers below the top surface i.e. in the core of sample. Hence, texture measurements were carried out on XY-MID and YZ planes as illustrated in Figure 2. The crystallographic texture observed at the XY-MID plane, near the sample center, represents the crystal orientation of 1 to 2 successively re-melted layers, as the employed layer thickness is $30 \mu \mathrm{m}$ and the penetration depth of copper k-alpha X-rays in copper is nearly $50 \mu \mathrm{m}$. Whereas, the texture obtained on the $\mathrm{YZ}$ plane represents the crystal orientation within more than 30 successive layers, as the beam size of the employed X-ray source was $1 \mathrm{~mm}$. Therefore, the latter texture measurement gives a representative image of the crystal orientation throughout the whole sample.

\subsection{1) Global texture beneath the top surfaces with $\langle 100\rangle$ texture parallel to BD}

Figure 7 shows texture measurements on three different planes within the same sample. The pole figures shown in Figure 7 (a) represents the texture obtained within the XY-TOP plane i.e. top melt pool layer. However, Figures 7 (b) and 7 (c) show the pole figures recorded for the XY-MID and YZ plane, respectively. They represent the texture developed due to multiple re-melting and heat exposure cycles in a horizontal and vertical section, respectively.

When SLM was performed using a laser power of $600 \mathrm{~W}$, a scan speed of $200 \mathrm{~mm} / \mathrm{s}$ and a hatch spacing of $0.09 \mathrm{~mm}$, a strong <100> texture parallel to SD and TD, as well as a weak $\langle 100\rangle$ texture parallel to BD was obtained on the top surface of the sample (XY-TOP). However, this pronounced $\langle 100\rangle$ texture parallel to all the three directions (SD, TD and BD) was eliminated during subsequent laser melting with a $90^{\circ}$ scan rotation between the layers and a random texture is obtained as shown in Figures 7 (b) and 7 (c). 


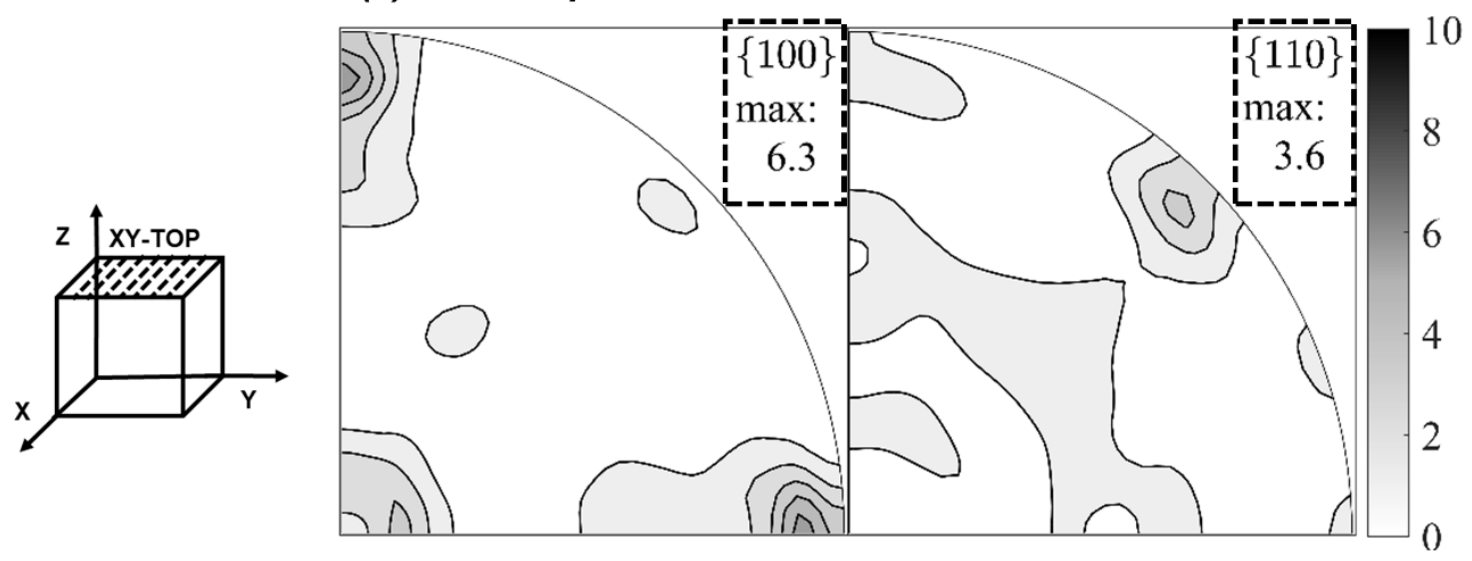

(b) XY-MID plane
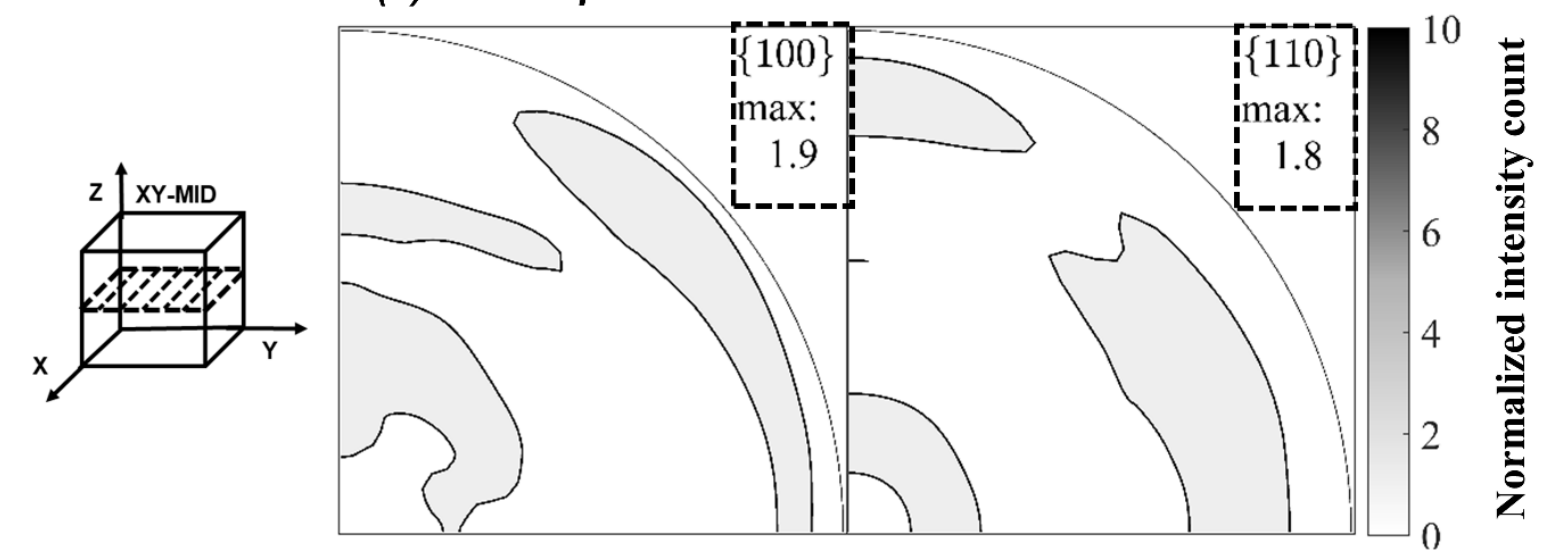

(c) YZ plane
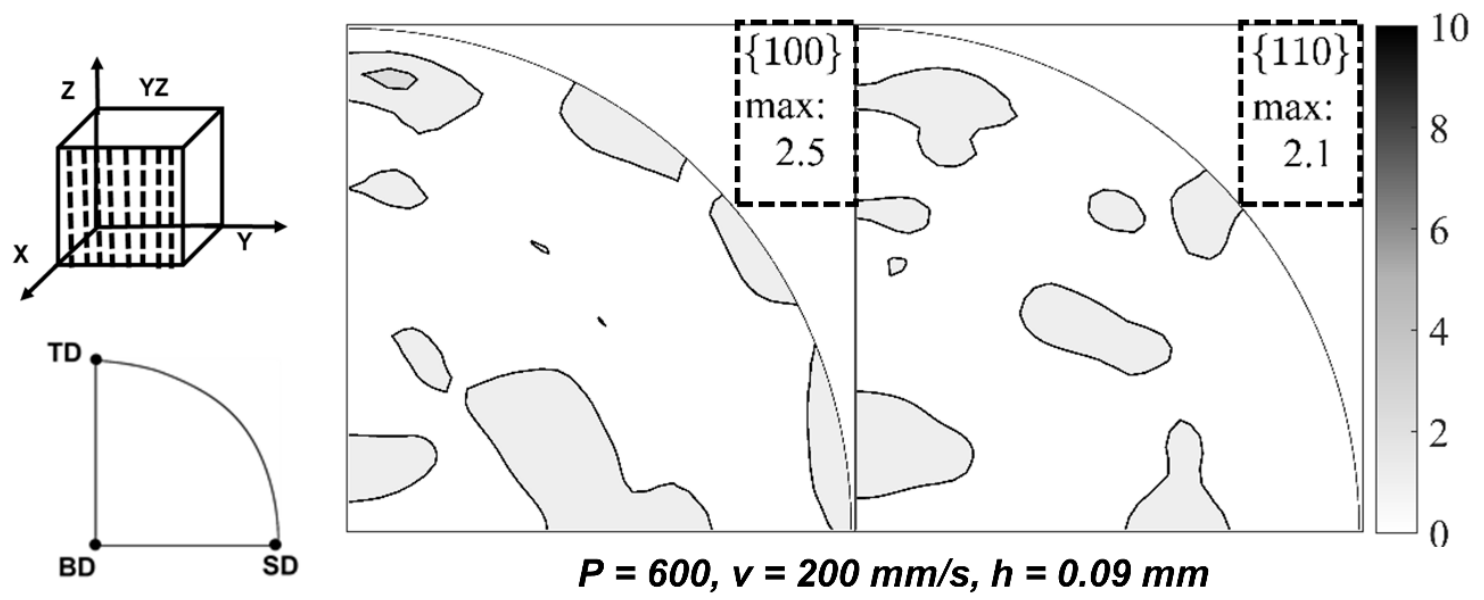

Figure 7: Influence of multiple re-melting and heat exposure cycles between the layers on texture development, for the sample processed at $P$ of $600 \mathrm{~W}, v$ of $200 \mathrm{~mm} / \mathrm{s}, h$ of $0.09 \mathrm{~mm}$. $\{100\}$ and $\{110\}$ pole figures are plotted for (a) top surface (XY-TOP), (b) horizontal section near center (XY-MID), (c) YZ plane. A strong $\langle 100\rangle$ texture developed in the top melt pool layer as shown in 7 (a) was eliminated during subsequent re-melting and heat exposure and nearly random texture is obtained in the bulk of the sample as shown in 7 (b) and 7 (c).

\subsection{2) Global texture beneath the top surfaces with $<110>$ texture parallel to BD}


When SLM was performed using a laser power of $800 \mathrm{~W}$, a scan speed of $400 \mathrm{~mm} / \mathrm{s}$ and a hatch spacing of $0.09 \mathrm{~mm}$, a strong <100> texture parallel to SD and TD and a weak $<110\rangle$ texture parallel to BD was generated as observed on the top surface of the sample (XYTOP). Figures 8 (a), 8 (b) and 8 (c) display the pole figures plotted for XY-TOP, XY-MID and YZ planes, respectively. Similar to the results for the samples that exhibited a strong $\langle 100\rangle$ texture at the top surface, the $\langle 110\rangle$ texture obtained parallel to BD in this case is also diminished due to multiple re-melting and heat exposure cycles caused by a $90^{\circ}$ scan rotation strategy employed between subsequent scan layers. 

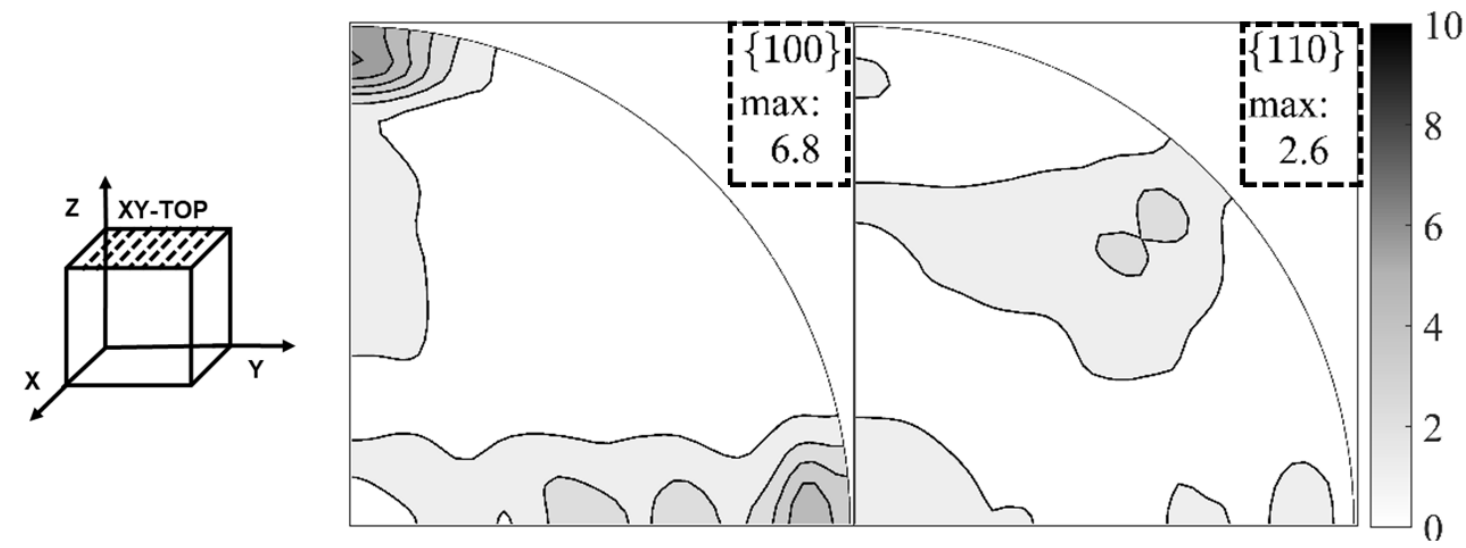

(b) XY-MID plane
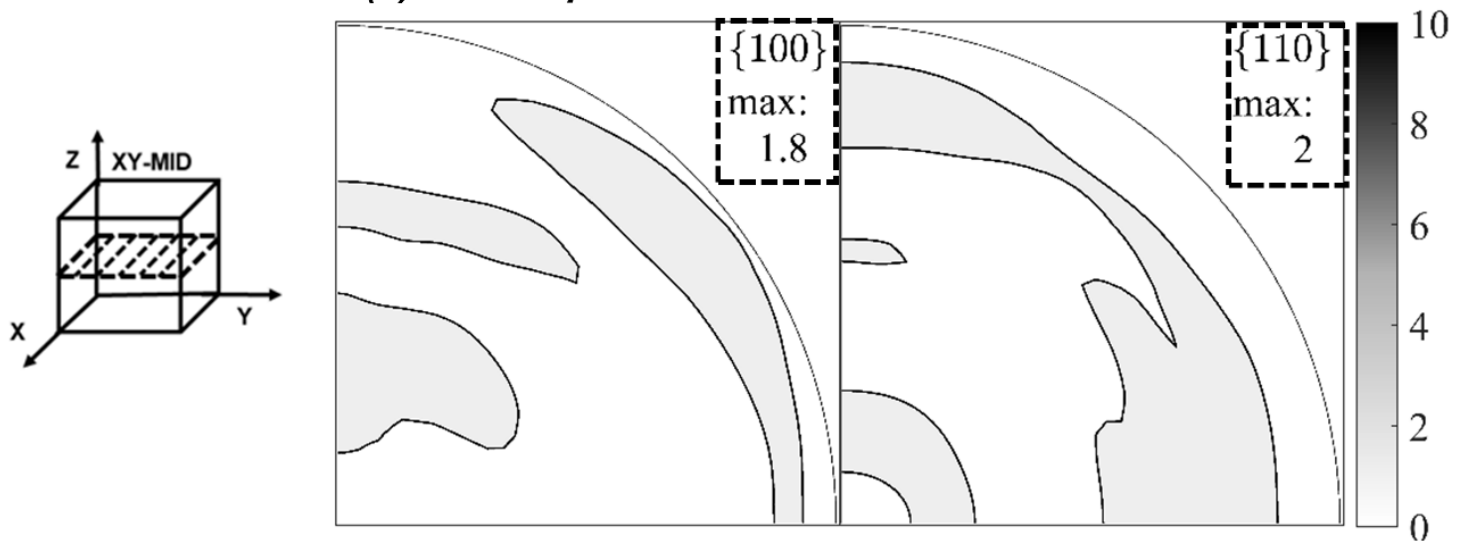

(c) YZ plane
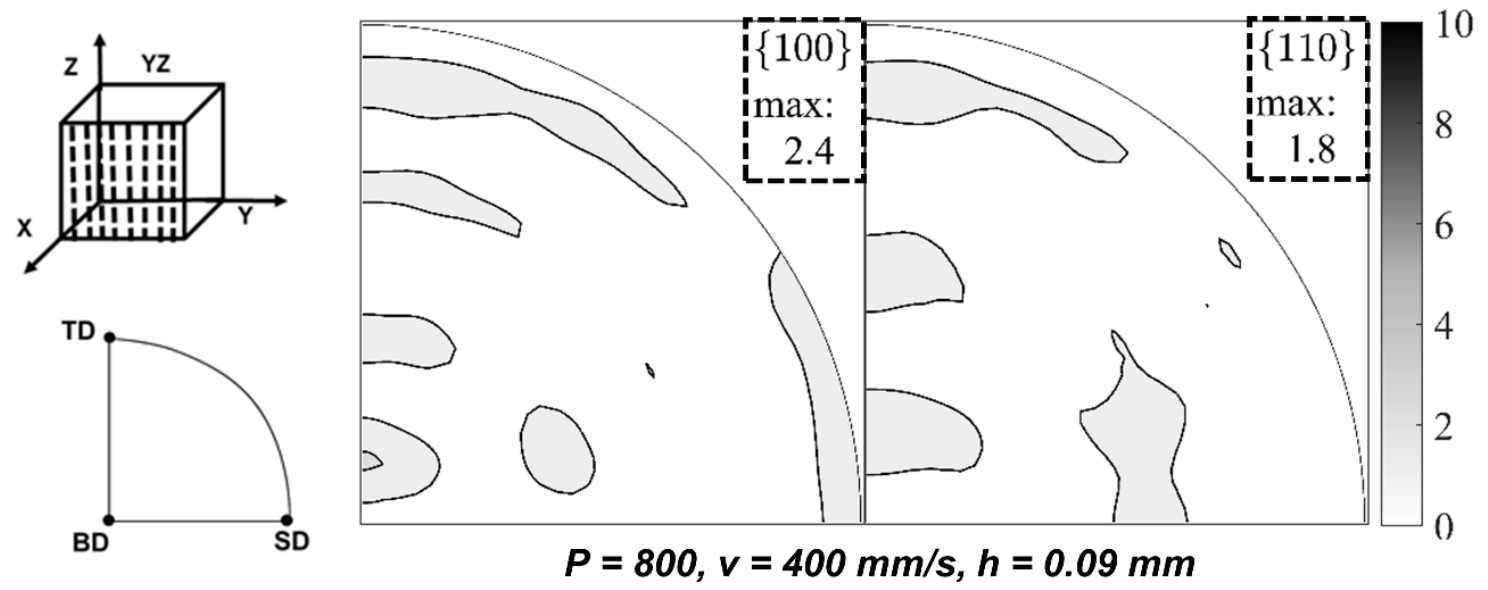

Figure 8: Influence of re-melting, heat exposure and change of temperature gradient due to $90^{\circ}$ scan rotation strategy between the layers on texture development for the sample processed with $P$ of $800 \mathrm{~W}, v$ of $400 \mathrm{~mm} / \mathrm{sec}, h$ of $0.09 \mathrm{~mm}$. $\{100\}$ and $\{110\}$ pole figures are plotted for (a) top surface (XY-TOP), (b) horizontal section near center (XY-MID), (c) YZ plane . A strong $<100>$ texture parallel to SD and TD and a weak $<110>$ texture parallel to BD is observed on the top surface as shown in 8 (a) is eliminated during subsequent remelting of layers with a $90^{\circ}$ scan rotation strategy and a nearly random texture is generated in the bulk of sample as shown in 8 (b) and 8 (c). 


\section{5) Electrical conductivity}

Since optimized SLM scan conditions were defined to process fully dense, crack-free and randomly oriented copper samples, the electrical conductivity of the SLM processed materials could be determined. Table 2 overviews the electrical conductivity values of SLM processed samples exhibiting stable sample geometries.

Table 2: Overview of laser parameters and obtained electrical conductivity results.

\begin{tabular}{llll}
\hline Laser parameter & $\begin{array}{l}\text { Energy Density } \\
\left(\mathbf{J} / \mathbf{m m}^{\mathbf{3}}\right)\end{array}$ & $\begin{array}{l}\text { Electrical } \\
\text { conductivity } \\
(\% \mathbf{O A C S})\end{array}$ & $\begin{array}{l}\text { Estimated } \\
\text { thermal } \\
\text { conductivity } \\
(\mathbf{W} /(\mathbf{m} . \mathbf{K}))\end{array}$ \\
\hline$P 800 \mathrm{~W}, v 400 \mathrm{~mm} / \mathrm{s}, h 0.09 \mathrm{~mm}$ & 741 & $88 \pm 2$ & $336 \pm 7$ \\
$P 800 \mathrm{~W}, v 400 \mathrm{~mm} / \mathrm{s}, h 0.07 \mathrm{~mm}$ & 952 & $86 \pm 3$ & $329 \pm 11$ \\
$P 600 \mathrm{~W}, v 200 \mathrm{~mm} / \mathrm{s}, h 0.09 \mathrm{~mm}$ & 1111 & $83 \pm 3$ & $317 \pm 12$ \\
\hline
\end{tabular}

The corresponding thermal conductivity values were estimated using the WiedemannFranz law (For, W-F ratio $(L)=2.2 \mathrm{E}-0.8 \mathrm{~W} \Omega / \mathrm{K}^{2}$ at $300 \mathrm{~K}$ ) (William and March, 1985). A maximum electrical conductivity of $88 \%$ IACS was obtained. Overall, all the samples possess very high electrical conductivities in the as built state.

\section{4) Discussion}

\section{1) Consequences of using high power lasers during SLM of highly reflective copper}

As the laser beam mainly interacts with a liquid melt pool during SLM, it can be expected that only a limited amount of laser light will be effectively absorbed by the SLM copper part and that a major amount is back reflected. Generally, the optical mirrors of an SLM machine are coated with a dielectric coating, engineered to minimize absorption at the laser's wavelength, nevertheless there is always a partial absorption. In the forward mode of operation, the laser spot is spread out over the beam steering mirrors to avoid excessive intensity levels. The beam is focused only after the f-theta lens onto the work plane. During SLM processing, however, there is always an amount of back reflection that travels back through the optical system (backward mode of operation) onto the optical mirror. Back reflections can create new focal points inside the optical system of the SLM machine. When such a focal point is situated on an optical mirror and the intensity of the back reflection exceeds the laser induced damage threshold (LIDT), damage of the optical coating will ensue. Indeed, after SLM processing of the aforementioned cube shaped copper samples, a small scratch was observed in the dielectric coating that covered the optical mirrors.

This indicated that the intensity of the laser back reflection exceeded the LIDT and thus damaged the optical coating. Once the coating is damaged, the reflectivity of the coating suffers, implying that the LIDT will lower. This will create a runaway effect, in which previous damage will lower the LIDT further and initiate new damage (Bloembergen, 1973). Moreover, processing of larger parts will require the use of longer scan tracks and larger scanning times 
per scanning layer. This contributes further to LIDT and enhances the runaway effect. Therefore, no large copper parts were processed by SLM during the current set of experiments.

After SLM of all cube shaped samples was finalized, the optical coating was intentionally exposed to 12 hours of back reflection originating from laser scan tracks that were generated on a bulk copper substrate with a reflectivity of $90 \%$ at the emitted wavelength of $1080 \mathrm{~nm}$. The resulting damage to the optical coating on the laser mirror is shown in Figure 9. Although it is not the primary objective of the current investigation, the aforementioned issue could be minimized by using alternative approaches to produce dense copper parts by SLM. The alternative approaches include i) Use of a green laser for which copper exhibits a higher optical absorption, ii) applying advanced multi variate ray tracings to avoid the occurrence of back focal points on the locations of the mirrors or iii) chemically modify the surface of the copper powder and melt so that the optical absorption at their surface is increased.

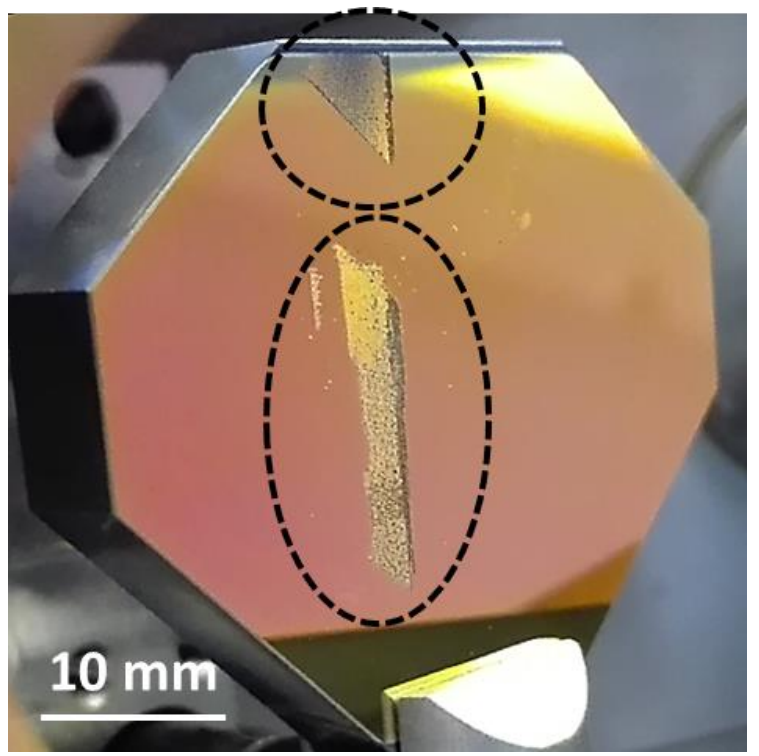

Figure 9: Image showing the damage to the optical mirror that was removed from the SLM machine after being exposed for $12 \mathrm{~h}$ to laser back reflection originating from laser scan tracks that were generated on a bulk copper substrate.

\section{2) The temperature gradient (G) and its interrelationship with solidification morphology}

Figure 10 (a) (Kou, 2003) shows the influence of temperature gradient (G) and growth rate $(\mathrm{R})$ on the solidification morphology. A planar solidification is realized at high $\mathrm{G}$ and low $\mathrm{R}$ whereas, a cellular or dendritic solidification is obtained at relatively lower $\mathrm{G}$ and higher $\mathrm{R}$. Figure 10 (b) (Kou, 2003) shows schematically the variation in orientation and magnitude of the $\mathrm{G}$ along the melt pool boundary. As the curvature of the melt pool is altered, $\mathrm{G}$ is also rotated and maintained perpendicular to the melt pool boundary. Furthermore, the $G$ is generally high at the melt pool fusion line and is low at melt pool centerline.

Accordingly, for the case of deep melt pools (obtained using high energy density), $\mathrm{G}$ is expected to be very high and oriented parallel to the top surface in the final melt pool layer. This results in the formation of planar solidification morphology as illustrated in Figure 5 (ac). Whereas, in case of elliptical melt pools (obtained using lower energy density), a lower 
value of $\mathrm{G}$ is expected resulting in formation of cellular morphology as shown in Figure 5 (df).

(a)

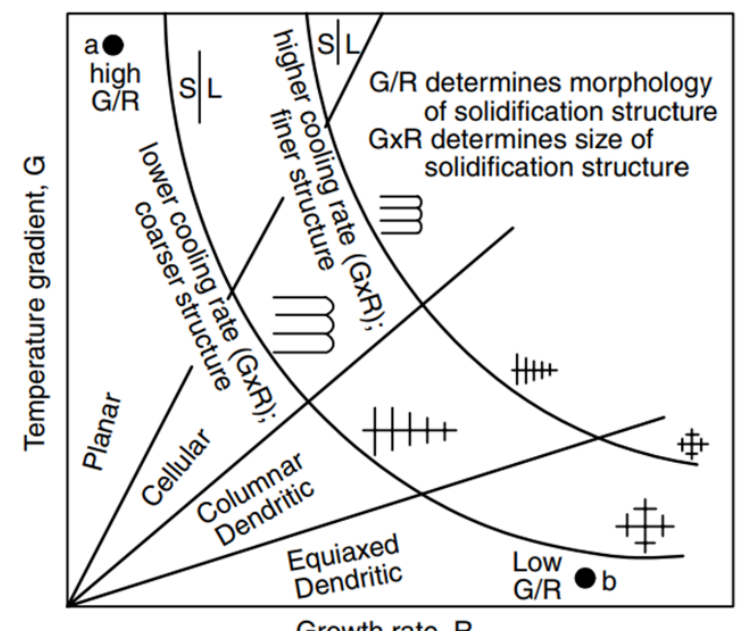

(b)

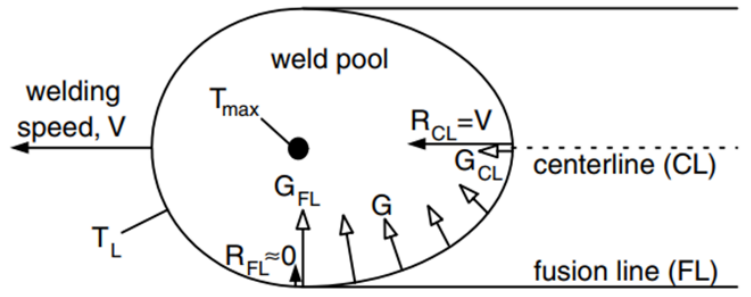

Figure 10: (a) Effect of temperature gradient $(G)$ and growth rate $(R)$ on solidification morphology, (b) Variation in temperature gradient $(\mathrm{G})$ and growth rate $(\mathrm{R})$ at melt pool boundary. The planar solidification morphology is achieved at high $\mathrm{G}$ whereas, a lower value of $G$ leads to formation of cellular or dendritic structure. The $G$ is always oriented perpendicular to the melt pool boundary and thus its orientation is changed along with the curvature of the melt pool. Reprinted from (Kou, 2003).

\section{3) SLM parameters and corresponding texture}

Table 3 summarizes the applied SLM scan parameters, resulting energy density values and their influence on the obtained crystallographic texture observed at the top surface of the SLM parts (XY-TOP). As the energy density increases, the texture type changes from a weak $\langle 110\rangle$ to a strong $\langle 100\rangle$ orientation parallel to BD. It can also be observed that, a variation of the hatch spacing between 0.07 and $0.09 \mathrm{~mm}$ has a limited influence on the texture generation when compared with the influence of the laser power and scan speed, within the selected set of parameters.

Table 3: Summary of laser scan parameters, corresponding energy density and resulting texture type and maximum texture intensity at XY-TOP.

\begin{tabular}{cccc}
\hline Laser parameter & $\begin{array}{c}\text { Energy density } \\
\left(\mathbf{J} / \mathbf{m m}^{\mathbf{3}}\right)\end{array}$ & $\begin{array}{c}\text { Texture type } \\
\text { parallel to } \\
\text { BD }\end{array}$ & $\begin{array}{c}\text { Max. texture } \\
\text { intensity } \\
\text { (Normalized } \\
\text { intensity count) }\end{array}$ \\
\hline$P 800 \mathrm{~W}, v 400 \mathrm{~mm} / \mathrm{s}, h 0.09 \mathrm{~mm}$ & 741 & $<110>$ & 2.6 \\
$P 800 \mathrm{~W}, v 400 \mathrm{~mm} / \mathrm{s}, h 0.07 \mathrm{~mm}$ & 952 & $<110>$ & 3.1 \\
$P 600 \mathrm{~W}, v 200 \mathrm{~mm} / \mathrm{s}, h 0.09 \mathrm{~mm}$ & 1111 & $<100>$ & 6.3 \\
$P 800 \mathrm{~W}, v 200 \mathrm{~mm} / \mathrm{s}, h 0.09 \mathrm{~mm}$ & 1481 & $<100>$ & 26 \\
\hline
\end{tabular}


The change in laser parameters causes a change in the energy density and thus, the shape and size of the melt pool is altered. The higher the applied energy density, the deeper the generated melt pool and vice versa. A high energy density is achieved, either by increasing the laser power and/or by lowering the scan speed and/or hatch spacing. Therefore, a high power, a low scan speed and a decreased hatch spacing would result in the formation of deeper melt pools, when observed perpendicular to scan direction (YZ plane). An elliptically shaped melt pool will be observed when looking at the top of the melt pool, parallel to scan direction (XYTOP plane). When, a low power, a high scan speed and a large hatch spacing are applied, the melt pool will be less deep and will appear broad and shallow as observed perpendicular to scan direction (YZ plane). The top view of such a melt pool will appear as an elongated ellipse or a conical or a teardrop shaped as illustrated in Figure 11.

\section{4) Melt pool geometry, solidification and texture development in the top surface}

Figure 11 schematically shows the top (XY-TOP) and YZ plane view of three different melt pool geometries (deep, elliptical and broad) associated with different laser scan parameters. Corresponding orientation of FCC unit cells, growing in $\langle 100\rangle$ direction parallel to the temperature gradient are also indicated for YZ plane. With increasing the scanning speed, the melt pool shape changes from the elliptical to an elongated ellipse or a conical or a teardrop shaped at the top surface. This can be visualized as a shape change from deep to elliptical or broad and shallow within YZ plane. Obtaining a broad and shallow melt pool geometry using a Gaussian laser beam at a high scan speed is very difficult. However, a top hat laser can produce broad and shallow melt pools. Thus, the schematic situation used to explain the texture evolution in the broad and shallow melt pool was not observed in case of pure copper. Nevertheless, (Montero-Sistiaga et al., 2018) demonstrated formation of broad melt pools for two FCC alloys namely 316L (Montero-Sistiaga et al., 2018) and Hastelloy X (MonteroSistiaga et al., 2019), by using a top hat laser.

Since $\langle 100\rangle$ is the easy growth direction in cubic metals, during the solidification of FCC metals, the grains with $\langle 100\rangle$ orientation are preferentially orientated parallel and opposite to the direction of the maximum temperature gradient $(\mathrm{G})$ due to competitive growth mechanism (Kou, 2003). Moreover, a change in size and shape of the melt pool causes a change in the orientation of the $\mathrm{G}$ and thus an alteration in the orientation of the $\langle 100\rangle$ oriented grains as shown in Figure 10 (b) and Figure 11). Consequently, the observed changes in $\langle 100\rangle$ or $<110>$ texture parallel to $\mathrm{BD}$, on the top surface can be explained using the melt pool geometries within the $\mathrm{YZ}$ plane.

In case where a deep melt pool is generated within $\mathrm{YZ}$ plane, the average temperature gradient and hence the $\langle 100\rangle$ growth direction in the upper part of the melt pool is oriented horizontally (parallel to the top surface). This orients the $\langle 001\rangle$ direction parallel to $\mathrm{BD}$, leading to a $<100>$ texture parallel to $\mathrm{BD}$ as shown in the corresponding inverse pole figure. Furthermore, the change in the melt pool geometry from deep to elliptical, which is accompanied by a reduction in the melt pool depth at YZ plane, rotates the average temperature gradient at an angle $\left(10-45^{\circ}\right)$ with respect to the top surface plane (see Figure 5 (f)). Consequently, the $\langle 100\rangle$ direction is oriented at a similar angle with respect to the top surface 
plane rendering a $\langle 110\rangle$ texture parallel to $\mathrm{BD}$ and a $\langle 100\rangle$ is oriented at an angle $10-45^{\circ}$ with respect to $\mathrm{BD}$ as shown in the respective inverse pole figure. Finally, if a broad and shallow melt pool is generated, the direction of the average temperature gradient is nearly perpendicular to the top surface and thus a $<100>$ texture can again be obtained parallel to BD.

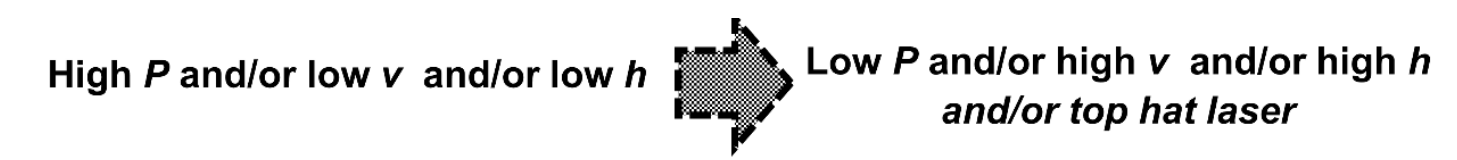

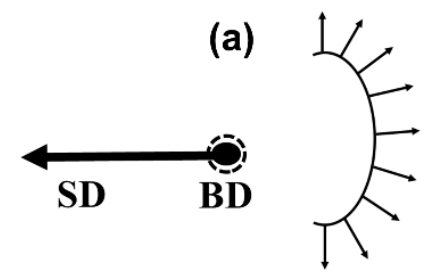

Elliptical melt

pool

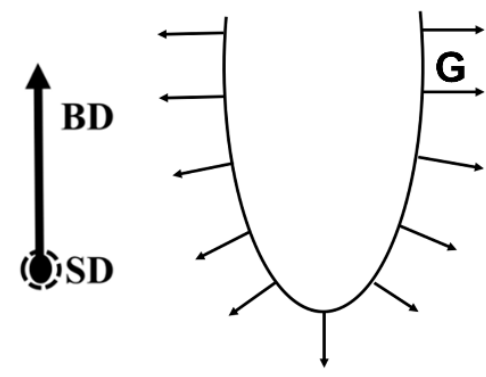

Deep melt pool
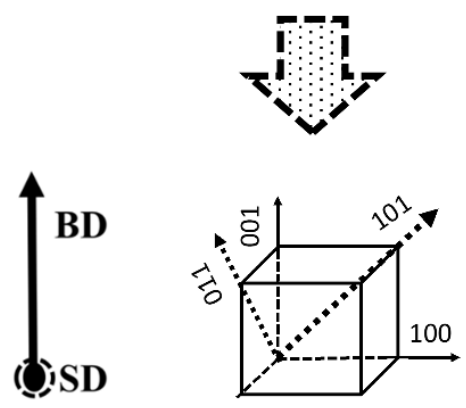

$<100>$ II to BD (b)

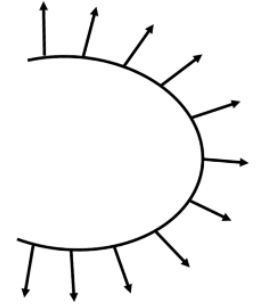

Elliptical melt pool

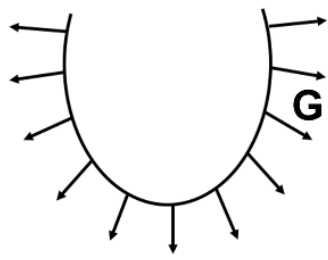

Elliptical melt pool (c)

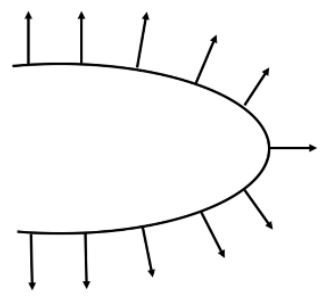

Conical/Teardrop melt

pool<smiles>CCC(C)C(C)C(C)C(C)C(C)C(C)C(C)C(C)C</smiles>

Broad and shallow melt

pool

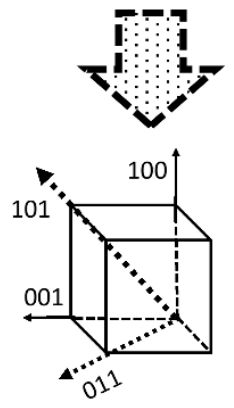

$<100>$ II to BD
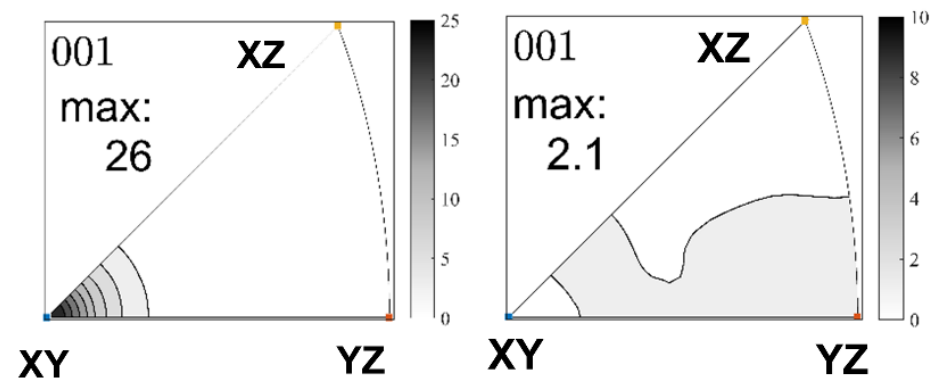

Figure 11: The schematic of melt pool geometries for top surface (XY-TOP) and YZ plane as well as the dominant orientation of the crystals leading to some typical inverse pole figures (as achieved in the present work). This figure shows (a) a deep melt pool on YZ plane results in a $\langle 100\rangle$ orientation parallel to $\mathrm{BD},(\mathrm{b})$ an elliptical melt pool on YZ plane rendering a weak $\langle 110\rangle$ texture parallel to BD and the $\langle 100\rangle$ orientations are inclined at an angle with 
top surface as shown in the inverse pole figure, (c) Broad and shallow melt pool at YZ plane (can be obtained at high laser scan speeds) giving a $<100>$ texture parallel to BD. (Note: XZ is a side surface similar to $\mathrm{YZ}$ plane but parallel to laser scan direction of the top melt pool layer).

The above paragraphs explain the changes in texture type and intensity from $\langle 100>$ and $<110\rangle$ parallel to BD. However, change in orientation of a $\langle 100\rangle$ texture is also observed when changing a scan speed from 200 to $400 \mathrm{~mm} / \mathrm{s}$ as shown in Figure 6 (c) and 7 (b) respectively. Sample processed with scan speed of $200 \mathrm{~mm} / \mathrm{s}$ shows a $<100\rangle$ orientated grains at angle of about $45^{\circ}$ between SD and TD and parallel to BD. This result can be explained using a modified schematic of a melt pool and solidification geometry obtained from (Kou, 2003) as shown in Figure 12. At low speed, the melt pool geometry is elliptical i.e. the melt pool has a curved surface and thus the maximum temperature gradient will also be curved (from melt pool boundary towards melt pool center) with respect to SD and TD. Therefore, the substructure in the columnar grains with $\langle 100\rangle$ easy growth directions is oriented at an angle with respect to SD and TD. Accordingly, the $\langle 100\rangle$ texture is also observed at similar angles between SD and TD as shown in Figure 12 (a).

When a scan speed of $400 \mathrm{~mm} / \mathrm{s}$ is applied the $<100>$ oriented texture disappears from the position between SD and TD. However, the $\langle 100\rangle$ oriented grains attain a new orientation parallel to SD and TD, which can be explained using Figure 12 (b). At high speed (or with increased speed) the shape of the melt pool changes from the elliptical to an elongated ellipse or a conical or a teardrop shape. As the heat transfer and solidification along the boundary of the melt pool is nearly straight compared to the curved shape obtained at low speed, the growth of the columnar grains is also straight line between the melt pool boundary and melt pool center i.e. between SD and TD. Thus, a $<100>$ texture is appeared along SD and TD. 
(a) Low speed
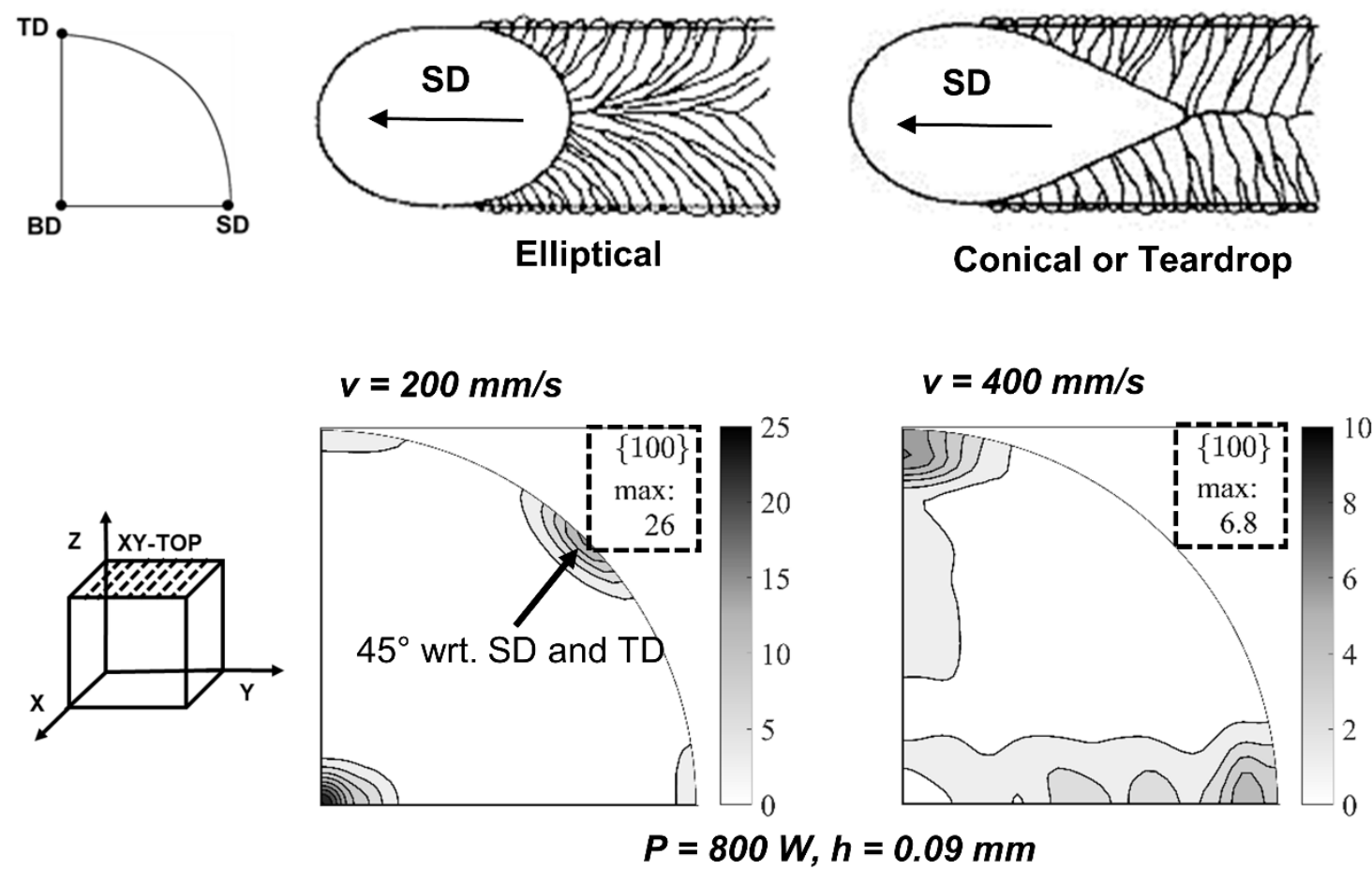

(b) High speed

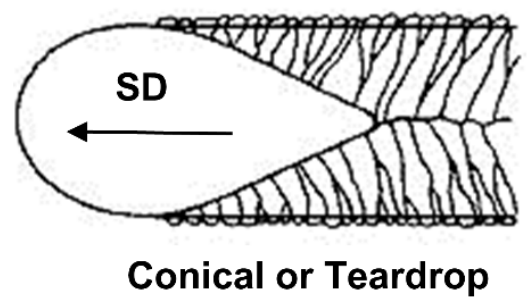

Conical or Teardrop

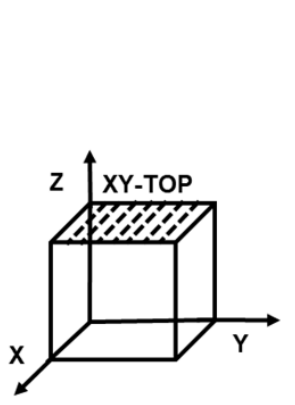

Figure 12: Schematic of melt pool geometry at (a) low and (b) high scan speeds showing influence of scan speed on the growth of columnar grains on top surface. The $\{100\}$ pole figures obtained in this research for samples processed at scan speed of 200 and $400 \mathrm{~mm} / \mathrm{s}$ are also shown to demonstrate the conforming changes in obtained texture. The schematic shown is reprinted from (Kou, 2003).

\section{5) Texture development in the bulk of sample and electrical conductivity}

Despite the strong texture observed in the top surface of the melt pool (measured on $\mathrm{XY}-\mathrm{TOP}$ ), the texture obtained in the bulk of sample (measured on YZ) is nearly random, irrespective of the applied SLM scan parameters. This can be associated with the successive re-melting and $90^{\circ}$ scan rotation strategy between the layers. The applied $90^{\circ}$ scan rotation strategy in the subsequent layers, rotates the average temperature gradient by $90^{\circ}$ with respect to the average temperature gradient of the previously melted layer. This causes successive remelting and regeneration of the crystallographic solidification texture of previous layers (as melt pool is over $100 \mu \mathrm{m}$ deep, but the layer thickness is only $30 \mu \mathrm{m})$. Moreover, copper as an excellent heat conductor of heat, intensifies the re-melting and crystal reorientations, leading to formation of nearly random texture in the bulk of sample.

Electrical conductivity being a physical property of copper, high electrical conductivity values are expected and reached for all the samples.

\section{5) Conclusion}

Dense and crack-free copper parts with excellent electrical properties were successfully SLMed using a high-power fiber laser. However, two factors currently limit its up scalability: i) the existence of a small SLM process window to obtain samples with a stable sample 
geometry and ii) high laser back reflection inducing damage to the dielectric coating on the optical mirror.

Analyzing the crystallographic texture of the SLM samples showed a relatively random crystal orientation for the middle (XY-MID) and YZ plane, despite the strong crystallographic texture at the top surface (XY-TOP). The crystallographic texture observed at the top surface could be explained by the solidification morphology, governed by the generated temperature gradients within the melt pools. The random texture within the bulk of the sample was explained by subsequent re-melting and altered heat gradient directions induced by the applied $90^{\circ}$ scan rotation strategy in between the subsequent layers.

The combination of SLM fabricated copper samples with high density, high purity and crystallographic isotropy resulted in a material with a very high electrical conductivity, reaching $88 \%$ of the International Annealed Copper Standard. The combination of nearly (crystallographic) isotropic and high electrical conductivity of this material and the SLM ability to manufacture geometrically complex parts, enables the application of SLM made pure copper as a functional material for heat and electricity transfer sectors.

\section{6) Acknowledgements}

The project was funded by the Flemish government agency for Innovation by Science and Technology (IWT) under the framework of Strategic Basic Research (SBO) project "Multimet". 


\section{7) References}

Aurubis, 2013. Heat Exchanger Material Facts \& Figures.

Bachmann, F., Hielscher, R., Schaeben, H., 2010. Texture Analysis with MTEX - Free and Open Source Software Toolbox. Solid State Phenom. 160, 63-68.

https://doi.org/10.4028/www.scientific.net/SSP.160.63

Bergström, D., 2008. The absorption of laser light by rough metal surfaces. Thesis. Luleå University of Technology.

Bloembergen, N., 1973. Role of Cracks, Pores, and Absorbing Inclusions on Laser Induced Damage Threshold at Surfaces of Transparent Dielectrics. Appl. Opt. 12, 661-664. https://doi.org/10.1364/AO.12.000661

Heussen, D., Meiners, W., 2017. Green Light for New 3D Printing Process, Fraunhofer Institute for laser technology (ILT) press release.

Ikeshoji, T.-T., Nakamura, K., Yonehara, M., Imai, K., Kyogoku, H., 2018. Selective Laser Melting of Pure Copper. JOM 70, 396-400. https://doi.org/10.1007/s11837-017-2695-X

Kou, S., 2003. Welding Metallurgy, Second Edition, Book. John Wiley \& Sons, Inc. https://doi.org/10.1002/0471434027

Lykov, P.A., Safonov, E.V., Akhmedianov, A.M., 2016. Selective Laser Melting of Copper. Mater. Sci. Forum 843, 284-288.

https://doi.org/10.4028/www.scientific.net/MSF.843.284

Montero-Sistiaga, M.L., Godino-Martinez, M., Boschmans, K., Kruth, J.P., Van Humbeeck, J., Vanmeensel, K., 2018. Microstructure evolution of 316L produced by HP-SLM (high power selective laser melting). Addit. Manuf. 23, 402-410.

https://doi.org/10.1016/j.addma.2018.08.028

Montero-Sistiaga, M.L., Pourbabak, S., Van Humbeeck, J., Schryvers, D., Vanmeensel, K., 2019. Microstructure and mechanical properties of Hastelloy X produced by HP-SLM (high power selective laser melting). Mater. Des. Jmade.

https://doi.org/10.1016/j.matdes.2019.107598

Neugebauer, R., Müller, B., Gebauer, M., Töppel, T., 2011. Additive manufacturing boosts efficiency of heat transfer components. Assem. Autom. 31, 344-347. https://doi.org/10.1108/01445151111172925

Prashanth, K.G., Scudino, S., Maity, T., Das, J., Eckert, J., 2017. Is the energy density a reliable parameter for materials synthesis by selective laser melting? Mater. Res. Lett. 5, 386-390. https://doi.org/10.1080/21663831.2017.1299808

Ryusuke, T., Yohei, O., Okamoto, A., Nakamoto, T., Sugahara, T., Shinomia, N., Takemura, M., Uchida, S., 2018. Copper alloy powder, method of producing additivelymanufactured article, and additvely-manufactured article. US2018/0111199 A1.

Thijs, L., 2014. Microstructure and Texture of Metal Parts Produced by Selective Laser Melting. KU Leuven.

Trevisan, F., Flaviana, C., Diego, M., Paolo, F., 2017. Selective laser melting of chemical pure copper powders, in: Euro PM2017. pp. 1-6. https://doi.org/10.1097/00000433198206000-00020

Van den Eynde, M., Strobbe, D., Verkinderen, O., Verbelen, L., Goderis, B., Kruth, J.-P., Van Puyvelde, P., 2018. Effect of thermal treatments on the laser sinterability of cryogenically milled polybutene-1. Mater. Des. 153, 15-23. 
https://doi.org/https://doi.org/10.1016/j.matdes.2018.04.072

William, J., March, N.H., 1985. Theoretical Solid State Physics. Courier Dover Publications. 


\section{List of figures}

Figure 1: Scanning Electron Microscopy (SEM) image of the copper powder that was used within this study, showing spherical powder particles along with some irregular particles and a small fraction of satellite particles attached to larger copper powder particles.

Figure 2: Schematic of texture measurement planes (a) top surface (XY-TOP), (b) horizontal section at center (XY-MID) and (c) vertical side surface - YZ plane. The measurement data for the $\mathrm{YZ}$ plane are rotated $90^{\circ}$ around the $\mathrm{Y}$ axis, in order to obtain $\mathrm{BD}$ parallel to $\mathrm{Z}$, similar to the case of XY-TOP and XY-MID planes.

Figure 3: (a) and (b) SEM images for the polished top surface (XY plane) and cross-section of the sample processed at low power and energy density of $300 \mathrm{~W}$ and $500 \mathrm{~J} / \mathrm{mm}^{3}$, respectively. The sample shows a high porosity content consequently, it was not useful for the texture measurements, (c) Visual impression of the fabricated cube shaped parts along with the corresponding SLM process parameters - $P$ in Watt, $h$ in $\mathrm{mm}$ and $v$ in $\mathrm{mm} / \mathrm{s}$, (d) Plot of obtained relative density $(\%)$ of parts with respect to applied energy densities $\left(\mathrm{J} / \mathrm{mm}^{3}\right)$. Parts with a stable geometry are marked with a circle.

Figure 4: SEM image of sample top surfaces (XY-TOP) processed with different SLM scan parameters. The laser scan direction is indicated by the white dashed arrows. All samples are dense, crack-free and have stable laser melt tracks.

Figure 5: (a), (b) and (c) Microstructures of the melt pool in the sample top layer showing mainly planar solidification morphology along with some cells oriented parallel to XY-TOP plane. These samples were fabricated using high energy densities. (d), (e) and (f) Microstructures of the samples processed at lower energy densities showing predominantly cellular morphology in the top layer melt pool. Most of the cells are oriented at an angle of 10$45^{\circ}$ relative to the top surface as shown in Figure (e) and (f) by white arrows. The maximum temperature gradient $(\mathrm{G})$ is indicated using black arrows.

Figure 6: The $\{100\}$ and $\{110\}$ pole figures showing the influence of different SLM scan parameters on texture development within the final melt pool layer. The respective scanning parameters are indicated on the top of each pole figure and texture measurements were performed on the top surface (XY-TOP). Note that Figure 6 (c) has a different scale bar, since it shows a much stronger texture. The black dashed arrow indicates the texture type parallel to building direction. It is either $\langle 100\rangle$ or $\langle 110\rangle$ parallel to the building direction depending on the employed laser setting.

Figure 7: Influence of multiple re-melting and heat exposure cycles between the layers on texture development, for the sample processed at $P$ of $600 \mathrm{~W}, v$ of $200 \mathrm{~mm} / \mathrm{s}, h$ of $0.09 \mathrm{~mm}$. $\{100\}$ and $\{110\}$ pole figures are plotted for (a) top surface (XY-TOP), (b) horizontal section near center (XY-MID), (c) YZ plane. A strong <100> texture developed in the top melt pool layer as shown in 7 (a) was eliminated during subsequent re-melting and heat exposure and nearly random texture is obtained in the bulk of the sample as shown in 7 (b) and 7 (c).

Figure 8: Influence of re-melting, heat exposure and change of temperature gradient due to $90^{\circ}$ scan rotation strategy between the layers on texture development for the sample processed with 
$P$ of $800 \mathrm{~W}, v$ of $400 \mathrm{~mm} / \mathrm{sec}, h$ of $0.09 \mathrm{~mm}$. $\{100\}$ and $\{110\}$ pole figures are plotted for (a) top surface (XY-TOP), (b) horizontal section near center (XY-MID), (c) YZ plane . A strong $<100\rangle$ texture parallel to SD and TD and a weak $<110>$ texture parallel to BD is observed on the top surface as shown in 8 (a) is eliminated during subsequent re-melting of layers with a $90^{\circ}$ scan rotation strategy and a nearly random texture is generated in the bulk of sample as shown in 8 (b) and 8 (c).

Figure 9: Image showing the damage to the optical mirror that was removed from the SLM machine after being exposed for $12 \mathrm{~h}$ to laser back reflection originating from laser scan tracks that were generated on a bulk copper substrate.

Figure 10: (a) Effect of temperature gradient $(\mathrm{G})$ and growth rate $(\mathrm{R})$ on solidification morphology, (b) Variation in temperature gradient $(\mathrm{G})$ and growth rate $(\mathrm{R})$ at melt pool boundary. The planar solidification morphology is achieved at high $\mathrm{G}$ whereas, a lower value of $\mathrm{G}$ leads to formation of cellular or dendritic structure. The $\mathrm{G}$ is always oriented perpendicular to the melt pool boundary and thus its orientation is changed along with the curvature of the melt pool. Reprinted from (Kou, 2003).

Figure 11: The schematic of melt pool geometries for top surface (XY-TOP) and YZ plane as well as the dominant orientation of the crystals leading to some typical inverse pole figures (as achieved in the present work). This figure shows (a) a deep melt pool on YZ plane results in a $<100\rangle$ orientation parallel to $\mathrm{BD}$, (b) an elliptical melt pool on $\mathrm{YZ}$ plane rendering a weak $\langle 110\rangle$ texture parallel to BD and the $\langle 100\rangle$ orientations are inclined at an angle with top surface as shown in the inverse pole figure, (c) Broad and shallow melt pool at YZ plane (can be obtained at high laser scan speeds) giving a $<100>$ texture parallel to BD. (Note: XZ is a side surface similar to $\mathrm{YZ}$ plane but parallel to laser scan direction of the top melt pool layer).

Figure 12: Schematic of melt pool geometry at (a) low and (b) high scan speeds showing influence of scan speed on the growth of columnar grains on top surface. The $\{100\}$ pole figures obtained in this research for samples processed at scan speed of 200 and $400 \mathrm{~mm} / \mathrm{s}$ are also shown to demonstrate the conforming changes in obtained texture. The schematic shown is reprinted from (Kou, 2003).

\section{List of tables}

Table 1: Powder characteristics of gas atomized copper powder used within this study.

Table 2: Overview of laser parameters and obtained electrical conductivity results.

Table 3: Summary of laser scan parameters, corresponding energy density and resulting texture type and maximum texture intensity at XY-TOP. 